\title{
Matrix-driven jamming dynamics mediates transition of ovarian cancer spheroids to stable morphologies
}

\author{
Tavishi Dutta , Jimpi Langthasa ${ }^{\mathrm{b}}$, Monica $\mathrm{U}^{\mathrm{b}}$, Prosenjit Sen ${ }^{\mathrm{a}^{*}}$, Ramray Bhat $\mathrm{t}^{\mathrm{b}^{*}}$ \\ a. Centre for Nanoscience and Engineering, Indian Institute of Science, India- 560012 \\ b. Department of Molecular Reproduction, Development and Genetics, Indian Institute of \\ Science, India- 560012 \\ *Prosenjit Sen, Ramray Bhat
}

Email: prosenjits@iisc.ac.in , ramray@iisc.ac.in

Author Contributions: T. Dutt., R. Bhat and P. Sen proposed the idea. T. Dutt, J. Langthasa, R. Bhat and P. Sen designed the research. T. Dutt performed microfluidic chip fabrication. T. Dutt and J. Langthasa executed research process, preliminary tests and formal analysis. T. Dutt and J. Langthasa validated and investigated experiments. Monica U. executed and analysed modelling and simulations. T. Dutt, R. Bhat and P. Sen wrote the manuscript. T. Dutt, J. Langthasa, Monica U., R. Bhat and P. Sen edited the manuscript. R. Bhat and P. Sen were responsible for supervision and funding acquisition.

Competing Interest Statement: There are no competing interests to declare.

Classification: Biological Sciences, Biophysics and Computational Biology.

Keywords: microfluidics, ovarian cancer spheroids, basement membrane, lumen, physics of cancer

\begin{abstract}
Metastatic progression of ovarian cancer through the peritoneal fluidic microenvironment is mediated predominantly by tumor cell clusters known as spheroids. Such spheroids also comprise the cells' extracellular matrix (ECM). Spheroids with lumen, often seen in malignant ascites, are associated with a basement membrane (BM)-like coat. Herein, we combine microfluidics with high- speed time-lapse videography and imaging analysis to investigate the consequences of lumen formation and the BM coat on the architecture and integrity of ovarian cancer spheroids providing architectural robustness to the transitory ovarian cancer metastatic niche within constrained flow spaces. Upon transit through spatially constrained environments, lumen-less 'moruloid' spheroids deform followed by an incomplete and temporally extended $(\sim 4.8 \mathrm{~s})$ recovery. The transit causes intercellular rearrangement during entry and shows susceptibility to cellular disintegration. On the other hand, lumen-containing 'blastuloid' spheroids exhibit minimal intra-spheroidal rearrangements. They exhibit a lower tendency for disintegration and quickly ( 1.5 s) recover their morphology upon exit. Significantly, removing the blastuloid BM through collagenase treatment reverts the mechanical behaviors of these spheroids to those typifying their moruloid counterparts. Simulations using an experimentally calibrated Cellular Potts model predict the requirement of higher intercellular adhesion for blastuloid behavior, which we confirm through increased E-cadherin expression in blastuloid
\end{abstract}


spheroids. Surprisingly, E-cadherin expression is dependent on the BM coat, elucidating the latter's role in enhancing cell-cell adhesion. Our results show how spheroidal matrix engenders lumen formation and higher intercellular adhesion, in turn providing architectural robustness of the transitory ovarian cancer metastatic niche within constrained flow spaces.

\section{Significance Statement}

Investigating the mechanical and biophysical properties of disseminated ovarian cancer spheroids is instrumental to our understanding of their metastatic progression. We take a microfluidics-based approach to investigate the biophysical contributions of a key spheroidal constituent: basement membrane-like extracellular matrix, which is typical to hollow 'blastuloid' spheroids, distinguishing them from the solid 'moruloid' spheroids. We observe using high-speed videography, imaging analysis and multiscale computational modeling that the basement membrane renders blastuloid spheroids less deformable and resistant to disintegration than moruloid spheroids. We trace this function to jamming of cells between the blastuloid lumen and basement membrane, and high intercellular adhesion.

\section{Introduction}

The peritoneal cavity of a patient suffering from advanced epithelial ovarian cancer is filled with disseminated multicellular aggregates, commonly known as spheroids (1). These spheroids colonize abdominal organs leading to metastasis (2). Metastasizing spheroidal cancer cells frequently become resistant to chemotherapeutic drugs necessitating a rigorous investigation of mechanisms underlying their formation and stability. Spheroids obtained by tapping the malignant ascites of ovarian cancer patients show heterogeneous morphologies: some exhibit a dysmorphic 'moruloid' (mulberry-like) phenotype, and others show smooth compacted surfaces and an internal lumen, giving them a 'blastuloid' appearance (3), (4). On the one hand, these diverse multicellular phenotypes could be aggregative consequences of phenotypically heterogeneous cell types that are shredded into the peritoneum. On the other hand, they could represent progressive stages of metastasis with moruloid phenotypes maturing into their blastuloid counterparts (5).

There is a burgeoning body of literature on biophysical investigations of tumorigenic cellular ensembles. Of these, most studies focus on the migrational dynamics of spheroidal or tumoroidal cells within stromal-like extracellular matrix (ECM) microenvironments (6),(7), (8). The dynamics of ECM confinement, local growth, and mesoscale processes such as adhesion and diffusive gradients of signaling and proteolysis are relevant to such contexts. In fluid microenvironments, the assembly of multicellular structures from suspended single cells likely employ distinct mechanisms (9), (10). Although elegant theoretical models have been constructed recently to explain dynamical structural transitions, technical difficulties of efficiently imaging floating clusters have allowed few biophysical characterizations of spheroids (11),(3). Notable experimental exceptions include efforts to mechanically analyze spheroids using microtweezers, wherein those constituted from breast cancer cells were found to be softer than from untransformed controls (12), and investigations using cavitational rheology to determine the cortical tension in spheroids of HEK293 cells (13). A pertinent study by Panwhar and coworkers recently describes a high throughput approach using virtual 
liquid-bound channels to show that the stiffness of multicellular spheroids is an order of magnitude lower than that of cells that constitute them (14). Although these investigations have not studied temporal topological transitions between multicellular morphologies, they lay the foundation for such studies within fluid microenvironments.

In this paper, we have probed the mechanical properties of ovarian cancer spheroids upon their traversal through a spatially constraining microfluidic channel. Previous studies have validated microfluidic approaches to be particularly useful in analyzing the mechanical properties of biomaterials, such as localized forces like traction, and their recovery dynamics (15), (16). We demonstrate that moruloid and blastuloid spheroids behave distinctly within the channel. Our biophysical investigations in combination with multiscale modelling reveal the importance of the ECM and cell adhesion in multicellular morphological transitions and provide insight into the sustained endurance of spheroids within the tortuous spatial landscape of the peritoneal cavity.

\section{Results}

\section{Absence of lumen leads to morphological deformation in spheroids upon constriction}

Moruloid (lumen-less) and blastuloid (lumen-containing) spheroids form in a temporally successive progression for the ovarian cancer cell line OVCAR3 upon suspended cultivation in low adherent conditions (Fig. 1A; red and white represent fluorescent signals for F-actin and DNA respectively). To investigate their mechanical properties, we subjected them to flow within a microfluidic channel that would constrain their movement in terms of size (Fig. 1B). Time lapse videography showed that moruloid spheroids emerged from the constrictive channel by assuming a shape that was deformed relative to their entry (Fig. 1C; Video S1), whereas the blastuloid spheroids progressively recovered their shape upon egress (Fig 1D; Video S2) (see also change in the minor axis as a function of emerging area (Fig. S1). The difference in egress characteristics were also observed for moruloid and blastuloid spheroids developed through cultivation of G1M2 ovarian cancer patient xenograft cells (Fig. S2, Videos S3 and S4). An important difference between moruloid and blastuloid spheroids involves the presence of a basement membrane (BM)-like ECM coat surrounding cells constituting the latter. This led us to also flow blastuloid spheroids after enzymatically debriding their BM coat using Type IV Collagenase (Fig. S3; green and white represent fluorescent signals for Type IV collagen and DNA, respectively and Video S5). The resultant exiting blastuloid spheroids showed shape deformation similar to the moruloid counterparts (Fig. 1E). We further quantified the deformation by measuring the aspect ratio of the leading edge of the spheroids as they exited the microfluidic channel. The aspect ratio was defined as

$$
\text { Aspect Ratio }(A R)=\frac{L}{w}
$$

where $w$ is the minor axis and $L$ is the major axis length of the protruding front-end of the spheroids (Fig. S4 A). These were quantified using a curve fitting equation representing that of an ellipse (Fig. S4 B) (see Materials and Methods for further details). The parameters returned from the fit were used to obtain the aspect ratio for each individual protruded spheroid, for different time-points during its exit. Change in the aspect ratios 
was tracked as a function of time for representative clusters of each subset (Fig. S4 C). The slopes were obtained by employing a simple linear fit on this curve. The mean slopes of aspect ratio change of blastuloid spheroids were observed to be significantly lower than that of moruloid clusters during channel exit. In addition, removal of BM in blastuloid spheroids increased the slope of their aspect ratio, suggesting continuous changes in the latter for blastuloid spheroids as they exited the channel $(p<0.001$; Fig. 1F).

We next traced the change in minor axis of spheroids normalized to their pre-entry values over longer periods of time: moruloid spheroids showed variable but incomplete extents of recovery with their minor axis plateauing within $~ 4.8 \mathrm{sec}$ (Fig. 1G). On the other hand, blastuloid spheroids completely recovered their minor axis within $1.5 \mathrm{sec}($ Fig. $1 \mathrm{H})$, but upon removal of BM showed incomplete and slower relaxation $(6.2 \mathrm{sec})$ (Fig. 1I). These observations suggested that moruloid spheroids deform more dramatically in comparison with blastuloid spheroids, and the BM contributes to this difference in ensemble mechanical behavior (Fig. 1J, Videos S6, S7 and S8).

\section{Constrictive traversal of blastuloid but not moruloid spheroids is size-autonomous}

In order to verify how the motility of the spheroids through constrictive spaces would depend on their size, we measured three time-metrics:

(i) Entry Time: defined as the time taken by the spheroid to fully enter into the channel and calculated as the difference between the times when the leading edge first touches the channel, and the rear edge of the cluster first touches the channel (Fig. 2A).

(ii) Travel Time: defined as the time taken by the cluster to move through the channel, before the leading edge leaves the channel and calculated as the difference between transit time and entry time (Fig. 2B).

(iii) Transit time: defined as the time a spheroid takes to pass through the channel and calculated as the difference between the times when the leading edge first touches the channel, and the rear edge of the cluster is just about to exit the channel (Fig. S5 A).

The mean entry and travel times for the moruloid spheroids (1.26 sec and $0.1 \mathrm{sec}$ ) were longer than that for blastuloid spheroids $(0.4 \mathrm{sec}$ and $0.06 \mathrm{sec})$ although the trend was not significant due to wider variation in the former (Fig. 2C and D; transit times showed a similar trend (Fig. S5 B). However, the mean times for the BM-removed blastuloid spheroids were significantly longer than that for their controls $(2.8 \mathrm{sec}$ and $0.14 \mathrm{sec}$, respectively: comparisons using one way ANOVA $p$ value $=0.002$ and 0.02 respectively) (Fig. 2C and D). We then sought to investigate the contribution of spheroidal size to their traversal. We observed that the distribution of points cognate to blastuloid spheroids was distinct from point distributions cognate to the moruloid and BM-removed blastuloid spheroids (centroids associated with the area-time distributions for moruloid and BMremoved blastuloid spheroids were closer to each other than to the counterpart for blastuloid spheroids Fig. 2E and F; K-L divergence values shown in Fig. 2G). In addition, entry and travel times correlated moderately with spheroid size for moruloid spheroids $\left(R^{2}\right.$ $=0.4$ for both), whereas the correlation was poor for untreated blastuloid spheroids $\left(R^{2}=\right.$ 0.01 and 0.02 , respectively) (Fig. S6). However, BM-removal restored the correlation of the time to the spheroid size during entry $\left(R^{2}=0.2\right)$ but not for travel through the channel $\left(R^{2}=0.008\right)$ (Table 2). We next asked if the increased time for entry and travel observed 
for moruloid spheroids was due to rearrangement in intercellular contacts as a result of travel through constrictive spaces.

\section{Intercellular positional variation occurs within moruloid spheroids during entry into channel}

To comprehend the flow of cells within spheroids when they traverse the channel, we used the PIVlab plugin in MATLAB to obtain color-coded plots showing the intensity of velocity gradients (Blue: low velocity; yellow: high velocity). Additionally, we annotated the figures with streamlines (white) and velocity vectors represented by arrows (black) to better picture the flow (17), (18). The velocity vectors were obtained from correlation peaks, which aligned with the direction of flow. Fig. 3 A-C show the flow maps obtained from analyzing the images in ImageJ for moruloid, blastuloid and BM-removed blastuloid spheroids respectively.

For all morphologies, the intensity of the vectors was higher within the channel (consistent with shorter travel times shown above) and the vectors aligned to form laminar streams (Fig. 3A-C). The differences were evident at the channel entry and exit. For the moruloid and BM-removed blastuloid spheroids, several vectors were misaligned in the streamlines that entered the channel leading to asymmetries at the upper and lower points of the channel (Fig. 3A and C). High-speed videos of the spheroids entering the channel showed considerable intercellular unjamming behavior in the lumen-less moruloid spheroids at the entry of the channel. This led to multidirectional motion for cells at the center and periphery of such spheroids at such spatial locales. For blastuloid spheroids, most of the vectors at entry were aligned with streamlines that entered the channel suggesting efficient alignment (Fig. 3B). This was quantifiably confirmed by measuring the mean angle of the PIV vectors for the three conditions before entering (Fig. 3D), when inside (Fig. 3E), and while coming out of the channels (Fig. 3F). Whereas the average angle between the vectors for moruloid and BM-removed blastuloid spheroids showed a wide divergence were compared at entry (interquartile range $=0.32$ and 0.16 , respectively), within the channel (interquartile range $=0.14$ and 0.08 , respectively) and at exit (interquartile range $=0.14$ and 0.11 , respectively), that for the blastuloid spheroids showed a narrower distribution which suggested a symmetric entry and expansion at exit (interquartile range $=0.06$ at entry, 0.04 in the channel and 0.02 at exit, respectively). This was consistent with a jammed lumen-depleting entry for blastuloid spheroids and an unjammed asymmetric entry and exit for the moruloid and BM-removed blastuloid spheroids.

\section{Basement membrane stabilizes blastuloid morphology under constrictive traversal}

To investigate if constrictive travel has a detrimental effect on spheroidal morphology, we probed for cell detachment or disintegration in clusters as they travelled through our channel. We found a greater disintegrating fraction in moruloid spheroids (0.364) than in blastuloid spheroids (0.058) (Fig. 4A; micrographs showing cell detachment in moruloid spheroids shown in Fig. 4B). Not only that, BM-removed blastuloid spheroids also showed a greater propensity for cell detachment during travel through the channel (disintegrating fraction being 0.463) (Fig. 4A; one way ANOVA $p=0.01$ ). The lumen and BM could combinedly lend a cushioning effect to the motion of the blastuloid clusters. Since spheroids vary in size, we also probed the effect of the latter on their propensity for disintegration. We also found that the blastuloid spheroids that did show cell detachment 
were considerably larger in size than their disintegration-exhibiting moruloid and ECMremoved counterparts (one way ANOVA $p<0.0001$; Fig. 4C). Larger clusters undergo larger strains, and hence are more prone to breakage (Videos S9, S10 and S11). This suggested that both BM and size-dependent strain play an important role in maintaining morphological stability with the former being dominant over the latter in its effect on the propensity for cell breakage. Our results with cell detachment led us to next investigate the effects of cell-cell adhesion on constrictive traversal of distinct morphologies.

\section{A multiscale model predicts lower cell adhesion in moruloid spheroids}

Multiscale computational models have been used with a great deal of success to simulate experimentally elucidated observations and verify whether multicellular phenotypes can indeed emerge through the empirical interactions $(19,20,21)$ Compucell3D represents one of the best-known simulation frameworks allowing an integration of Cellular Potts model-based solving of a Hamiltonian for contact energies between cellular constituents with PDE solvers of molecular constituents (22),(7).

We therefore constructed multicellular models for the moruloid and blastuloid spheroids, which were driven through a constrictive channel within the simulation space, similar to our experimental setup. The input parameters that were tunable within such simulations were the contact energies (inverse of adhesion strength) between individual digital cells, and between digital cells and their surrounding medium. We confirmed that upon exiting the simulated constrictive channel space 'digital moruloid' spheroids showed a sustained deformation and intercellular rearrangement (through observed intermixing of peripheral (blue) and central (green) cells of the spheroids) (Fig. 5A and Video S12). On the other hand, the digital blastuloid spheroids recovered their shape rapidly upon exit (Fig. 5B and Video S13). Insets of exiting digital moruloid and blastuloid spheroids showed intercellular rearrangements in the former but not the latter, showing consistence with our experimental findings (Fig. 5A and B insets to the right). Consistent with these observations, the aspect ratio of egressing digital moruloid morphologies when normalized to entry aspect ratio was found to be significantly higher than that for digital blastuloid counterparts (unpaired t-test $\mathrm{p}<0.0001$; Fig. $5 \mathrm{C}$ ). In fact, at lower values of intercellular adhesion, we also observed cell detachment and disintegration for both digital moruloid (Fig. 5D and Video S14) and blastuloid spheroids (Fig. 5E and Video S15). In agreement with the experimental observations, we found greater cell detachment in the case of digital moruloid spheroids $(46.67 \%)$ than with the digital blastuloid spheroids (20\%) (Fig. 5F). Upon increasing intercellular adhesion, a decrease in normalized aspect ratio was observed for digital moruloid spheroids, suggesting that cell-cell adhesion may regulate the unjamming-tojamming transition of multicellular ensembles (one-way ANOVA $p<0.0001$; Fig. 5G).

Interepithelial adhesion is principally mediated through adherens junctions, established using homodimeric interactions between transmembrane cell adhesion molecules such as E-cadherin (23). The expression of E-cadherin has been shown to be under the regulation of Laminin 111, a principal constituent of epithelial basement membrane (BM) matrix (24). Our earlier demonstration of BM expression being characteristic of blastuloid ovarian cancer spheroids, our observation of a lesser propensity of blastuloid spheroids to show cell detachment and our theoretical prediction of an association of higher intercellular adhesion with lower aspect ratio led us to ask whether the blastuloid spheroids expressed comparatively greater levels of E-cadherin. Using fluorescent immunocytochemistry, we 
observed greater transmembrane signals for E-Cadherin in blastuloid spheroids compared with moruloid spheroids, suggesting that E-cadherin driven by BM expression, contributes to the distinct rheological behaviors of moruloid and blastuloid ovarian cancer spheroids (Fig. 5H). Upon BM-removal, the intercellular staining of E-cadherin in blastuloid spheroids was found to be weaker than control untreated spheroids suggesting a cross regulation between cell-cell and cell-ECM adhesion contributes to the architectural integrity of blastuloid spheroids (Fig. 5I).

\section{Discussion}

At the heart of our study is an important observation: that the same cancer cell can form distinct multicellular architectures with unique mechanical properties. A spheroid that is constituted entirely of cells with no internal lumen exhibits deformability upon strain due to spatially constrictive travel. In addition, their shape recovery time-scales are far slower than their deformation timescales indicating irreversible changes in their mechanical structure indicative of plastic behavior. Under strain they also show cellular disintegration. On the other hand, a lumen containing spheroid shows a jammed cellular organization with a low propensity for cellular detachment. Post deformation, their recovery time-scales are much faster and complete suggesting their morphology undergoes little change under constriction. Although this could be indicative of an elastic behavior of materials, their shorter entry times suggests that blastuloid spheroids are able to maintain their morphology by contextually reducing their luminal volume during entry. In fact, initial attempts to computationally model the blastuloid spheroids as impermeable elastic spheres resulted in longer entry times in simulations as well. This was mitigated upon simulating a dynamic deflation-inflation behavior of the digital blastuloid spheroids during traversal. The kinetics of lumen reinflation of these spheroids upon exiting the constriction suggests that the lumen acts as a dynamical volume whose magnitude is a function of the strain faced by the spheroid. Furthermore, the similarity between the behavior of blastuloid spheroids upon BM removal and moruloid spheroids can be understood by how the BM is now shown to mediate two crucial morphogenetic characteristics of blastuloid spheroids: maintenance of lumen and high intercellular adhesion. The lumen-less spheroids on the other hand are morphogenetically labile, owing to a loss of the external BM and lower intercellular adhesion. Therefore, the kinetics of regaining a more spheroidal shape occurs on a longer scale than for blastuloid spheroids. Further, the recovery is incomplete as is indicated by our PIV analysis, due to an irreversibly altered arrangement of the cells constituting them.

As moruloid spheroids differentiate into blastuloid spheroids, the BM induces a cellular transition from an unjammed to a jammed state (25),(26). It has not escaped our attention that another transition occurs concurrently: a mesenchymal to epithelial transition (27),(28). The moruloid spheroids are associated with fibronectin expression, whereas we observe strong expression of E-cadherin in the blastuloid spheroids. Fibronectin and Ecadherin are classical markers of mesenchymal and epithelial states (29). Moreover, BM is also associated with the epithelial state of tissues (30). In addition, whereas a tendency to detach from a multicellular mass strongly characterizes a mesenchymal state, higher intercellular adhesion is typical of epithelial one (31). Taken together, the blastuloidmoruloid shift corresponds to a mesenchymal-to-epithelial transition (MET). Fredberg and colleagues have explored whether the transition between unjammed to jammed cell behavior and that between mesenchymal to epithelial states are conceptually non- 
congruent across biological examples (31). Our study seeks to bridge the two frameworks by showcasing how a limited set of proteins shift multicellular states both in terms of their polarity and motility.

Do spheroids encounter such spatially restricted spaces within the peritoneal cavity? Apart from the classical epiploic foramen that connects the lesser and greater sac, there are several peritoneal fossae, wherein spheroids may potentially be subjected to constriction (32). Moreover, the peritoneal spaces are dynamic owing to constant motion within the surrounding organs, such as peristasis. Such spatial restrictions could impinge on the rheologies of diverse spheroidal morphologies and enhance the spectrum of their behaviors: cell detaching from the moruloid spheroids are better suited to micrometastatic colonization, whereas the jammed stable blastuloid spheroids could represent a niche suited for longer survival within the ascites. This is seen through the prolific accumulation of the cancer cells within the ascites of ovarian cancer patients.

Our study throws up engaging questions. On a mechanistic level, what is responsible for dynamical behavior of the blastuloid spheroids: the lumen, the BM or the higher intercellular adhesion? Given that all these processes are entangled phenomenologically, it is not easy to dissect the exact contribution of each of these to the ensemble properties. BM debridement and dysregulation of E-cadherin through genetic approaches would be employed in a temporally controlled manner in the future to unravel the molecular mechanisms of intraspheroidal cell jamming. Multicellular ensembles have now been proposed to have greater potential for metastatic colonization than their unicellular counterparts. Could such potential be explained by their ability to withstand strain because of robustness to disintegration even in narrow peritoneal spaces? A broader generalization of our study to other cancers that employ both transperitoneal and vascular route of dissemination would shed light on the ubiquitous influence of rheological regimes on disseminated cancer cell behavior.

\section{Materials and Methods}

\section{Cell culture}

Ovarian cancer cell lines used in this study are OVCAR3 and G1M2 (patient derived xenograft line). These cell lines were maintained in Dulbecco's Modified Eagle's Medium - high glucose (AL007A; HiMedia) and Roswell Park Memorial Institute medium (AL162A; HiMedia) supplemented with 10-20\% FBS and recommended antibiotics in a humidified atmosphere of $95 \%$ air and $5 \% \mathrm{CO} 2$ at $37^{\circ} \mathrm{C}$.

\section{Spheroid culture and collagenase treatment}

Spheroid were cultured in defined medium for 1 day to 7 days in suspension. Medium composition: DMEM: F12 (1:1) (HiMedia AT140) supplemented with $0.5 \mathrm{\mu g} / \mathrm{ml}$ hydrocortisone (Sigma-Aldrich, H0888), $250 \mathrm{ng} / \mathrm{ml}$ insulin (Sigma-Aldrich, I6634), 2.6 $\mathrm{ng} / \mathrm{ml}$ sodium selenite (Sigma-Aldrich, S5261)), $27.3 \mathrm{pg} / \mathrm{ml}$ estradiol (Sigma-Aldrich, E2758), $5 \mu \mathrm{g} / \mathrm{ml}$ prolactin (Sigma-Aldrich L6520, $10 \mu \mathrm{g} / \mathrm{ml}$ transferrin (Sigma-Aldrich, T3309). $1.5 \times 10^{5}$ cells were seeded in polyHEMA coated $35 \mathrm{~mm}$ dishes for the experiment. Spheroids were collected from the cultures by centrifugation at $12000 \mathrm{rpm}$ for 
5 min. Collagenolysis of blastuloid spheroids was done by incubating with $600 \mathrm{U}$ collagenase IV (17104-019 Gibco) for 24 hrs.

\section{Immunocytochemistry and Imaging}

Spheroids were collected after centrifugation at $12000 \mathrm{rpm}$ and fixed using $3.7 \%$ formaldehyde (24005; Thermo Fisher Scientific) at $4^{\circ} \mathrm{C}$ for $20 \mathrm{~min}$. After one wash with PBS, the fixed spheroids were resuspended in PBS and put 10-20 $\mu$ of spheroids suspension to eight well chambered cover glass followed by placing on a dry bath at $37^{\circ} \mathrm{C}$ for 15-30 min for drying. Spheroids were then permeabilized using $0.5 \%$ Triton $X-100$ (MB031; HiMedia) for $2 \mathrm{~h}$ at RT. Using 3\% BSA (MB083; HiMedia) prepared in $0.1 \%$ Triton X-100 solution blocking was achieved at RT incubated for 45 minutes. Primary antibody against E-cadherin (24E10 Cell Signaling Technology) was incubated overnight at $4^{\circ} \mathrm{C}$ which was followed by washes using $0.1 \%$ TritonX-100 in PBS $(5 \min \times 3)$. Secondary antibody was incubated at RT for $2 \mathrm{~h}$ under dark conditions. DAPI (D1306; Thermo Fisher Scientific) was added to the samples and incubated for 15 minutes. Three washes were given after secondary antibody incubation step and after addition of DAPI. Images were captured in 20X and 40X using a Carl Zeiss LSM880 laser confocal microscope. Images were processed and analyzed using ZEN Lite software.

\section{Ellipse fitting algorithm for aspect ratio calculation}

To quantify the mechanical response of the spheroids, we opted to use Aspect Ratio of their ejection as a metric. It is obtained by fitting an ellipse to the protruding semi-circular shape coming out of the channel, for 4 different time points - front when the cluster is $25 \%$ exuding out of the channel to when it is almost $75 \%$, on the verge of complete escape.

The ellipse fitting is done using a MATLAB code, to find out the major and minor axes of the hence obtained ellipse. Using those values, we calculated the aspect ratios of the $\begin{array}{llll}\text { protruding } & \text { end } & \text { (Fig. } & \text { B). }\end{array}$ (https://www.mathworks.com/matlabcentral/fileexchange/15125-fitellipse-m).

\section{Modeling framework}

Compucell3D (CC3D) is a problem-solving environment based on the lattice-based GGH (Glazier-Graner-Hogeweg) model or CPM (Cellular Potts model) that was designed to model collective behavior of active matter (33). This is done by calculating the Hamiltonian energy function at each simulation step. In the simulation lattice, each cell is represented by rectangular Euclidean lattice sites or pixels that share the same cell ID. The model evolves at each Monte Carlo Step (MCS), which consists of index-copy attempts of each pixel in the cell lattice. Calculation of the Hamiltonian $(\mathrm{H})$ determines the allowed configuration and behavior of cells at each MCS.

$$
\begin{aligned}
H= & \sum_{\mathrm{i}, \mathrm{j} \text { neighbours }} J\left(\tau\left(\sigma_{i}\right), \tau\left(\sigma_{j}\right)\right)\left(1-\delta\left(\sigma_{i}, \sigma_{j}\right)\right)+\sum_{\sigma}\left[\lambda_{\text {vol }}\left(v\left(\sigma_{i}\right)-V\left(\sigma_{i}\right)\right)^{2}\right]+ \\
& \sum_{\sigma}\left[\lambda_{\text {surf }}\left(s\left(\sigma_{i}\right)-S\left(\sigma_{i}\right)\right)^{2}\right]+H_{E P}
\end{aligned}
$$

The Hamiltonian used in our model has two main contributors, which are affected by different properties of the cells. The first term in the energy function is the sum over all neighboring pairs of lattice sites $i$ and $j$ with associated contact energies $(\mathrm{J})$ between the pair of cells indexed at those $i$ and $j$. In this term, $i, j$ denotes pixel index, $\sigma$ denotes the 
cell index or ID, and t denotes cell-type. The $\delta$ function ensures that only the $\sigma_{i} \neq \sigma_{j}$ terms are calculated ( $i, j$ belonging to the same cell will not be considered). Contact energies are symmetric in nature $\left[\left(\tau\left(\sigma_{i}\right), \tau\left(\sigma_{j}\right)\right)=J\left(\tau\left(\sigma_{j}\right), \tau\left(\sigma_{i}\right)\right)\right]$. The contact energy between the two cells is considered inversely proportional to the adhesion between the two cells. The second term in the equation is a function of the volume constraint on the cell. $\lambda_{\text {vol }}(\sigma)$ denotes the inverse compressibility of the cell, $v(\sigma)$ is the number of pixels in the cell (volume), and $V_{t}(\sigma)$ is the cell's target volume. The third term in the equation is a function of the surface area constraint on the cell, as the cells have fixed amounts of the cell membrane. For the cell $\sigma, \lambda_{\text {surf }}(\sigma)$ denotes the inverse membrane compressibility of the cell, $s(\sigma)$ is the surface area of the cell, and $S(\sigma)$ is the cell's target surface area. The fourth term in the equation corresponds to the external potential applied to the center of mass of the cells to cause directional motion. $\Delta H_{E P}=-\overrightarrow{F_{\sigma(l)}} \cdot \overrightarrow{r_{l j}}$ is the Hamiltonian for the external potential for a given MCS. For a cell $\sigma$, during an index copy attempt from $i$ to $j$, the force vector is $\overrightarrow{F_{\sigma(l)}}$ And the distance between the pixels $i$ and $j$ is $\overrightarrow{r_{l j}}$. The product of these two vectors along the right direction will lead to energy minimization and result in the movement of the cell along that direction.

The acceptation or rejection of the index copy attempt from pixel $i$ to $j$ depends on the change in the Hamiltonian $(\Delta H)$ due to the change in energy after the index copy attempt. When $\Delta \boldsymbol{H} \leq \mathbf{0}$, the associated index-copy attempt will be successful, and the target pixels will be updated. So, the success probability is $P=1$. When $\Delta \boldsymbol{H} \geq \mathbf{0}$, the associated indexcopy attempt will be successful following the Boltzmann probability function, with a probability of $P=e^{-\left(\frac{\Delta H}{T m}\right)}$ and it will be unsuccessful with a probability of $P^{\prime}=1-P$. In the Boltzmann probability function, $\Delta H$ represents the calculated change in the overall Hamiltonian of the system between the system configuration at previous MCS and a specific system configuration at the current MCS. $T_{m}$ relates to the effective membrane fluctuation for the cell and is kept at $T_{m}=10$ in all the simulations.

A default dynamical algorithm known as modified Metropolis dynamics with Boltzmann acceptance function is used at each MCS to move the system towards a low-energy configuration as MCS increases. The term $\mathrm{T}_{\mathrm{m}}$ can be considered temperature or magnitude of effective membrane fluctuations. Random movements of the pixels leading to different transition probabilities at each MCS mimics the stochasticity present in biological systems.

\section{Model components:}

We use a $450^{\star} 200^{*} 1$-pixel square lattice with a non-periodic boundary for all the simulations. Any model element required to participate through MCS pixel-copy attempts must be assigned a cell type. This model consists of 4 different cell types: Chamber, Peripheral cells and Core cells, and the Medium. All cells are of dimension $5^{\star} 5^{\star} 1$ in size, except for the core of the moruloid spheroids, of size $35^{\star} 35^{\star} 1$. In an initial configuration, the chamber cells mimic the microfluidic chamber in the experimental setup, with the width to length aspect ratio maintained. These cells are "frozen" in the lattice, meaning the motility of the chamber cells is entirely restricted, and the movement of these cells to other lattice positions is not possible. The peripheral cells and the core cells make up the 36celled spheroid, with the peripheral cells forming the outer layer and the core cells forming the rest of the spheroid. The lattices with no assigned cell type or, in other words, the free spaces are assigned as cell type 'medium' as a default by the CompuCell3D algorithm. 
Contact energies (Differential Adhesion): CompuCell3D requires setting interactions between all cell types in the model in terms of contact energies. Contact energy is inversely related to cell-cell adhesion. Higher contact energy between two cell types implies a higher contribution to the effective energy and lower probability of adhesion of the two cells. Since we have 4 different cell types, 10 different contact energy values must be assigned. These values were assigned based on literature survey(33)(34). The contact energy values between Core-Core, Peripheral-Peripheral, and Core-Peripheral were considered input variables to observe the effect of morphology on the transition through the chamber.

External Potential: External potential was applied on the core and peripheral cells along the negative $x$-axis to enable movement of the spheroid through the chamber. After standardizing through parameter sensitisation, the value of the force vector was fixed at 6 for all simulations.

Compressibility: The compressibility of the core cells is different in the moruloid and the blastuloid spheroids to model the lumen in blastuloid spheroids. In the moruloid spheroids, the cells have higher inverse membrane compressibility fixed at 5 , similar to the peripheral cells, making the cells stiffer. The core cell in the blastuloid spheroids, made to mimic the lumen, has lower inverse membrane compressibility fixed at 0.5 to make them more compressible and fluid-like.

Modelling deflation and inflation of blastuloid spheroids: The blastuloid spheroids were modelled to deflate when met resistance while entering the narrow chamber. The velocity of the blastuloid spheroids reduces drastically when it meets resistance while entering the chamber, and the core of the spheroids were modelled to shrink in size every time the velocity of the spheroid dropped considerably below the baseline velocity. The baseline velocity of these spheroids, averaged over a frequency of 100 MCS, was considered as the velocity at which they moved in the free space due to the constant force acting on them by means of the external potential. When the velocity of the core of the spheroids reduced to less than half its baseline velocity, the target volume and target surface area of the core of the spheroids was reduced, thereby mimicking deflation of the blastuloid spheroid. Similarly, on exiting the narrow chamber, the spheroid no longer experiences the resistance and inflates. When the velocity of the core of the spheroids increases to twice its baseline velocity, the target volume and target surface area of the core was increased, to model the inflation of the spheroids (https://github.com/MonicaUrd/Spheroid tunnel resistance simulations.git).

\section{Microfluidic Chip Fabrication}

A Piranha cleaned 4" Silicon wafer is spin coated with SU-2035 (Microchem) negative photoresist at $3000 \mathrm{rpm}$ for 35 seconds to obtain a thickness of $75 \mu \mathrm{m}$. After which the wafer undergoes a soft bake for 3 mins at $65^{\circ} \mathrm{C}$ followed by 7 mins at $95^{\circ} \mathrm{C}$. Then it is UV

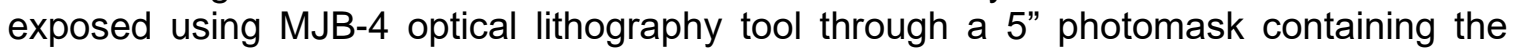
micropatterns. A post-exposure bake is done for 2 mins at $65^{\circ} \mathrm{C}$ followed by 6 mins at $95^{\circ} \mathrm{C}$. The wafer is then developed using SU-8 developer for 15 mins, followed by an IPA wash. It is coated with Teflon followed by a hard-bake for 45 mins at $150^{\circ} \mathrm{C}$.

The pattern is transferred to a glass slide using soft lithography. PDMS is mixed with a curing agent in 10:1 ratio and poured on to the wafer with a ring to seal the edges. It is desiccated in vacuum for around 5 mins to remove any settled bubbles. After that it is 
heated for 45 mins on $110^{\circ} \mathrm{C}$, and the hardened mold is peeled off once its cooled. Holes are punched for inlets and outlets using a $1 \mathrm{~mm}$ biopsy punch, following which the PDMS block is plasma bonded to an IPA cleaned glass slide after 1 min of treatment. The device is heated for 25 mins at $110^{\circ} \mathrm{C}$ to create a permanent bond.

\section{Experimental Setup}

The sample is loaded into the device using a $\mathrm{BD} 1 \mathrm{ml}$ syringe and microtubing. Using Chemyx Fusion high pressure pump, it is withdrawn (to avoid settling of clusters), at a rate of $1000 \mu \mathrm{l} / \mathrm{hr}$. The flow is visualized and recorded using a high-speed camera at $1000 \mathrm{fps}$, with 10x magnification lens. PFV-4 is used to view and analyse the recorded videos.

\section{Acknowledgments}

We would like to acknowledge Shahid Hussain for help with spheroid preparation. This work was supported by the Wellcome Trust/DBT India Alliance Fellowship grant (IA/I/17/2/503312) awarded to $R$ Bhat. $R$ Bhat would additionally acknowledge support from the Department of Biotechnology, India (DBT) (BT/PR27952/INF/22/212/2018 and BT/PR21962/NNT/28/1233/2017), and the Institute of Eminence grant (IE/CARE-190319). P Sen acknowledges DBT and MEITY. J Langthasa and T Dutt acknowledges Indian Institute of Science (IISc) for fellowship. MU acknowledges KVPY for the student scholarship (X08010248).

\section{References:}

1. E. Lengyel, Ovarian cancer development and metastasis. Am. J. Pathol. 177, 1053-1064 (2010).

2. K. Shield, M. L. Ackland, N. Ahmed, G. E. Rice, Multicellular spheroids in ovarian cancer metastases: Biology and pathology. Gynecol. Oncol. 113, 143-148 (2009).

3. J. Langthasa, et al., Extracellular matrix mediates moruloid-blastuloid morphodynamics in malignant ovarian spheroids. Life Sci. Alliance 4 (2021).

4. C. Koike, et al., Solid stress facilitates spheroid formation: potential involvement of hyaluronan. Br. J. Cancer 86, 947-953 (2002).

5. A. Goyeneche, et al., The Capacity of High-Grade Serous Ovarian Cancer Cells to Form Multicellular Structures Spontaneously along Disease Progression Correlates with Their Orthotopic Tumorigenicity in Immunosuppressed Mice. Cancers (Basel). 12 (2020).

6. K. S. Kopanska, Y. Alcheikh, R. Staneva, D. Vignjevic, T. Betz, Tensile Forces Originating from Cancer Spheroids Facilitate Tumor Invasion. PLoS One 11, e0156442 (2016).

7. D. Pally, et al., Heterogeneity in 2,6-Linked Sialic Acids Potentiates Invasion of Breast Cancer Epithelia. ACS Cent. Sci. 7, 110-125 (2021). 
8. A. J. McKenzie, et al., The mechanical microenvironment regulates ovarian cancer cell morphology, migration, and spheroid disaggregation. Sci. Rep. 8, 7228 (2018).

9. C. Duclut, N. Sarkar, J. Prost, F. Jülicher, Fluid pumping and active flexoelectricity can promote lumen nucleation in cell assemblies. Proc. Natl. Acad. Sci. 116, 19264-19273 (2019).

10. K. M. Burleson, et al., Ovarian carcinoma ascites spheroids adhere to extracellular matrix components and mesothelial cell monolayers. Gynecol. Oncol. 93, 170-181 (2004).

11. C. Duclut, J. Prost, F. Jülicher, Hydraulic and electric control of cell spheroids. Proc. Natl. Acad. Sci. 118 (2021).

12. D. Jaiswal, et al., Stiffness analysis of 3D spheroids using microtweezers. PLoS One 12, e0188346 (2017).

13. A. Blumlein, N. Williams, J. J. McManus, The mechanical properties of individual cell spheroids. Sci. Rep. 7, 7346 (2017).

14. M. H. Panhwar, et al., High-throughput cell and spheroid mechanics in virtual fluidic channels. Nat. Commun. 11, 2190 (2020).

15. K. D. Nyberg, et al., Quantitative Deformability Cytometry: Rapid, Calibrated Measurements of Cell Mechanical Properties. Biophys. J. 113, 1574-1584 (2017).

16. D. N. Breslauer, P. J. Lee, L. P. Lee, Microfluidics-based systems biology. Mol. Biosyst. 2, 97-112 (2006).

17. A. R. Khopkar, et al., Gas-Liquid Flow Generated by a Pitched-Blade Turbine: Particle Image Velocimetry Measurements and Computational Fluid Dynamics Simulations. Ind. Eng. Chem. Res. 42, 5318-5332 (2003).

18. S. Cai, J. Liang, Q. Gao, C. Xu, R. Wei, Particle Image Velocimetry Based on a Deep Learning Motion Estimator. IEEE Trans. Instrum. Meas. 69, 3538-3554 (2020).

19. N. J. Popławski, A. Shirinifard, M. Swat, J. A. Glazier, Simulation of single-species bacterial-biofilm growth using the Glazier-Graner-Hogeweg model and the CompuCell3D modeling environment. Math. Biosci. Eng. 5, 355-388 (2008).

20. O. Ilina, et al., Cell-cell adhesion and 3D matrix confinement determine jamming transitions in breast cancer invasion. Nat. Cell Biol. 22, 1103-1115 (2020).

21. A. E. Pelling, Y. Li, W. Shi, J. K. Gimzewski, Nanoscale visualization and characterization of Myxococcus xanthus cells with atomic force microscopy. Proc. Natl. Acad. Sci. U. S. A. 102, 6484-6489 (2005).

22. D. Pramanik, M. K. Jolly, R. Bhat, Matrix adhesion and remodeling diversifies modes of cancer invasion across spatial scales. J. Theor. Biol. 524, 110733 (2021). 
23. A. Hartsock, W. J. Nelson, Adherens and tight junctions: structure, function and connections to the actin cytoskeleton. Biochim. Biophys. Acta 1778, 660-669 (2008).

24. G. Benton, E. Crooke, J. George, Laminin-1 induces E-cadherin expression in 3dimensional cultured breast cancer cells by inhibiting DNA methyltransferase 1 and reversing promoter methylation status. FASEB J. 23, 3884-3895 (2009).

25. W. Kang, et al., A novel jamming phase diagram links tumor invasion to nonequilibrium phase separation. iScience 24, 103252 (2021).

26. K. Saitoh, T. Hatano, A. Ikeda, B. P. Tighe, Stress Relaxation above and below the Jamming Transition. Phys. Rev. Lett. 124, 118001 (2020).

27. E. Theveneau, R. Mayor, Neural crest migration: interplay between chemorepellents, chemoattractants, contact inhibition, epithelial-mesenchymal transition, and collective cell migration. Wiley Interdiscip. Rev. Dev. Biol. 1, 435445 (2012).

28. Y. Bernardi, P. H. Strobl-Mazzulla, What we can learn from embryos to understand the mesenchymal-to-epithelial transition in tumor progression. Biochem. J. 478, $1809-1825$ (2021).

29. E. Nowak, I. Bednarek, Aspects of the Epigenetic Regulation of EMT Related to Cancer Metastasis. Cells 10 (2021).

30. A. Pozzi, P. D. Yurchenco, R. V lozzo, The nature and biology of basement membranes. Matrix Biol. 57-58, 1-11 (2017).

31. G. F. Le Bras, K. J. Taubenslag, C. D. Andl, The regulation of cell-cell adhesion during epithelial-mesenchymal transition, motility and tumor progression. Cell Adhes. \&amp; Migr. 6, 365-373 (2012).

32. J. M. Thomas, K. Van Fossen, Anatomy, Abdomen and Pelvis, Foramen of Winslow (Omental, Epiploic) (StatPearls Publishing, Treasure Island (FL), 2021).

33. M. H. Swat, et al., Multi-scale modeling of tissues using CompuCell3D. Methods Cell Biol. 110, 325-366 (2012).

34. M. P. Steinkamp, et al., Ovarian tumor attachment, invasion, and vascularization reflect unique microenvironments in the peritoneum: insights from xenograft and mathematical models. Front. Oncol. 3, 97 (2013). 
bioRxiv preprint doi: https://doi.org/10.1101/2022.02.09.479678; this version posted February 9, 2022. The copyright holder for this preprint (which was not certified by peer review) is the author/funder, who has granted bioRxiv a license to display the preprint in perpetuity. It is made available under aCC-BY-NC-ND 4.0 International license.

A
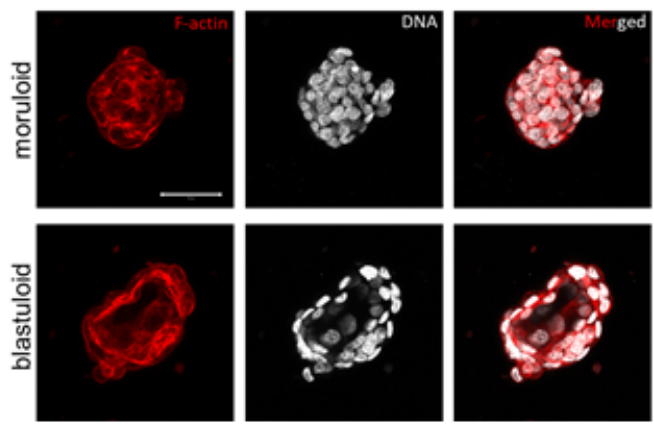

C

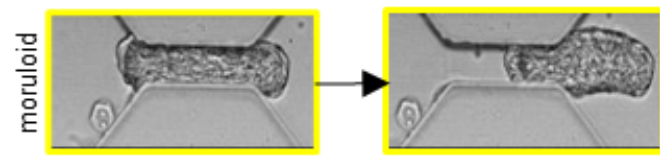

D
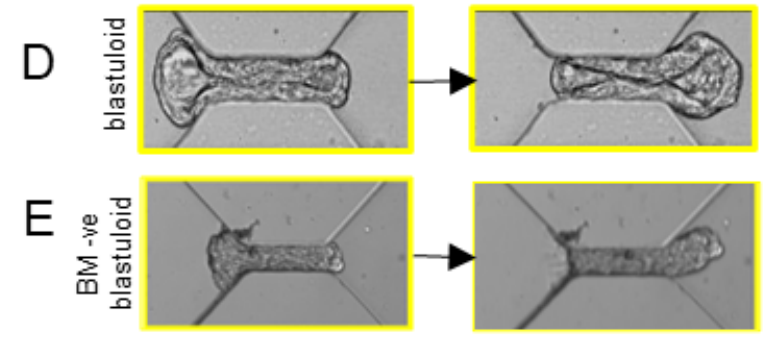

G

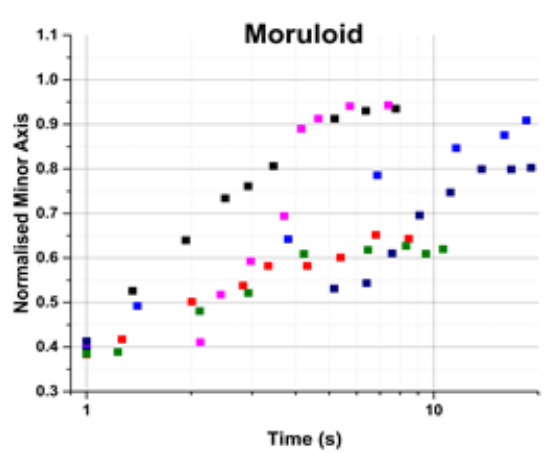

I

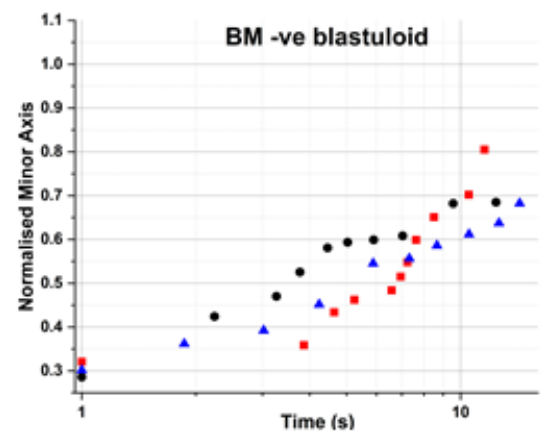

B

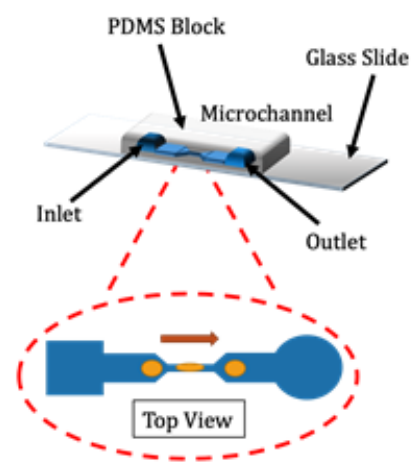

F

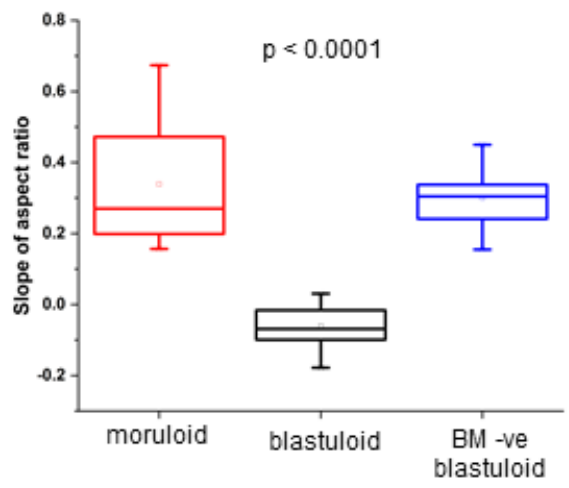

$\mathrm{H}$

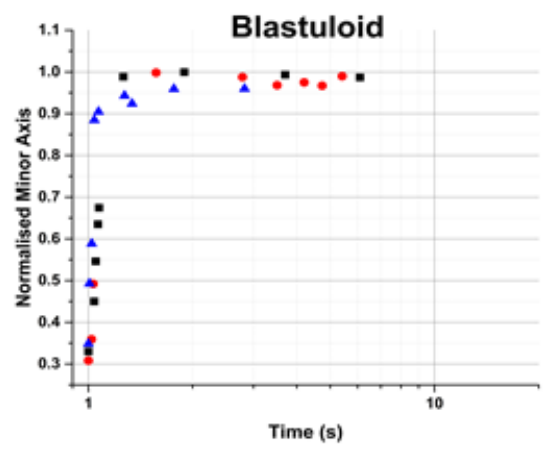

$J$

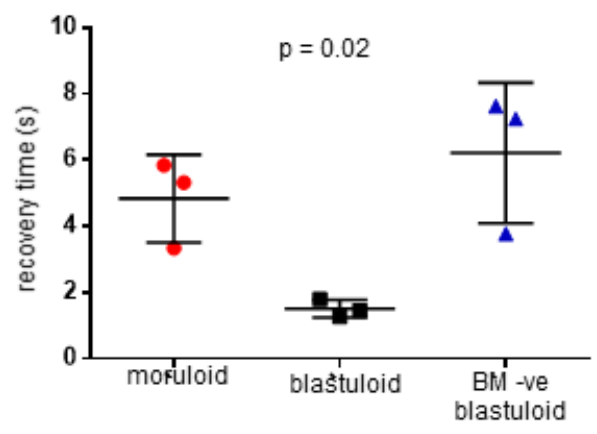




\section{Figure 1: Blastuloid spheroids relax efficiently and rapidly upon exit from constrictive travel.}

(A) Laser confocal micrographs of moruloid (top) and blastuloid (bottom) OVCAR3 spheroids showing maximum intensity projections of the fluorescence values representing F-actin (phalloidin; red) and DNA (DAPI; white). (B) Schematic depiction of a microfluidic chip used for performing the flow experiments. (C-E) Snapshots of high-speed camera videos for moruloid (C), blastuloid (D) and BM removed blastuloid spheroids (E) as they pass through a microfluidic channel (see also Video S1, S2 and S5). (F) Box-plot representing slopes of aspect ratios of moruloid, blastuloid, and BM-removed blastuloid clusters which are calculated using ellipse-fitting for the leading edge of the cluster as it exits the channel (see also Fig. S4 C). One way ANOVA with post hoc Tukey's comparison used to compute statistical significance $(p<0.0001)$. (G-I) Representative traces showing change in minor axis of exited $(\mathrm{G})$ moruloid $(\mathrm{H})$ blastuloid and $(\mathrm{I})$ BM-removed blastuloid spheroid as a function of time. (J) Scatter graph showing the minimum recovery time for moruloid, blastuloid, and BM-removed blastuloid spheroids that have exited the constrictive channel (see also Video S6, S7 and S8). One way ANOVA with post hoc Tukey's comparison used to compute statistical significance $(p=0.02)$. Error bars represent mean+/- SEM. Scale bars $=50 \mu \mathrm{m}$. 
A

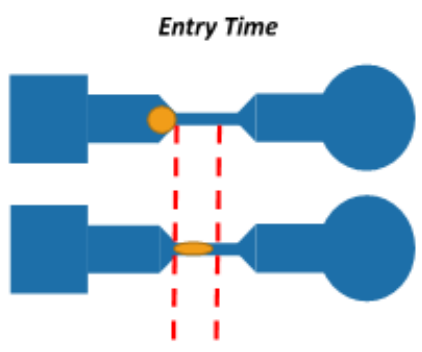

$\mathrm{dt}=\mathrm{te} 2$-te1

C

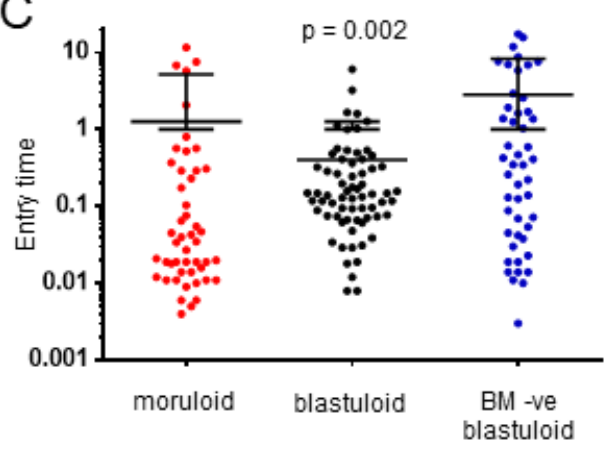

$E$

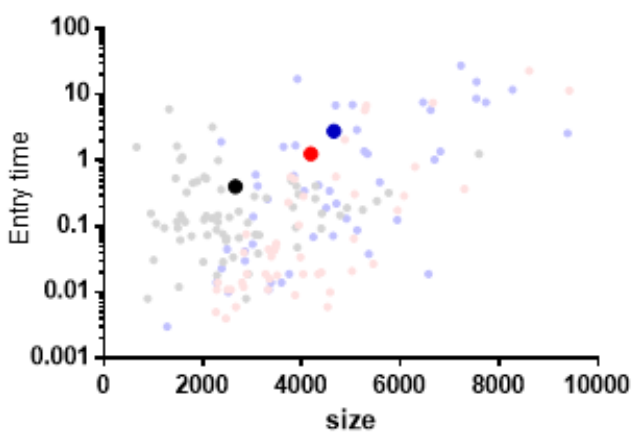

$B$
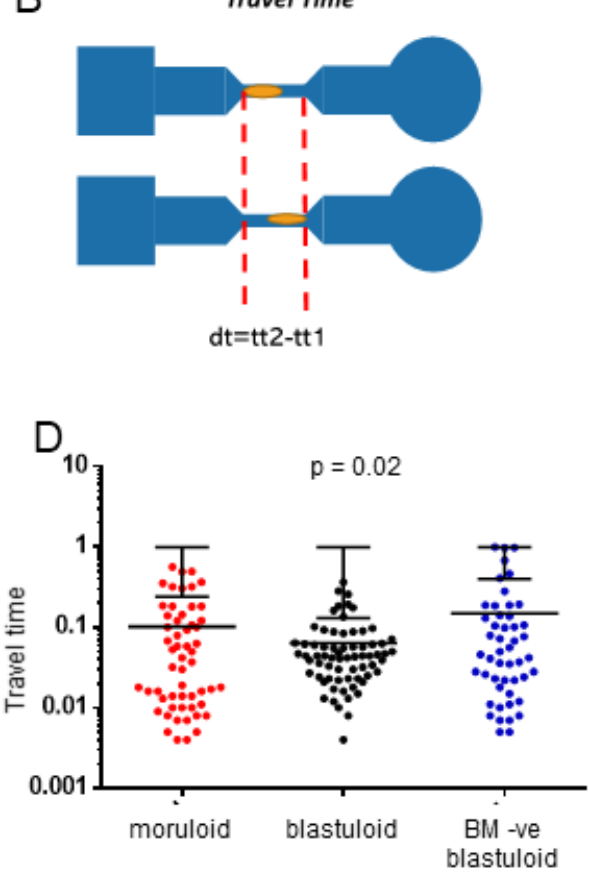

$\mathrm{F}$

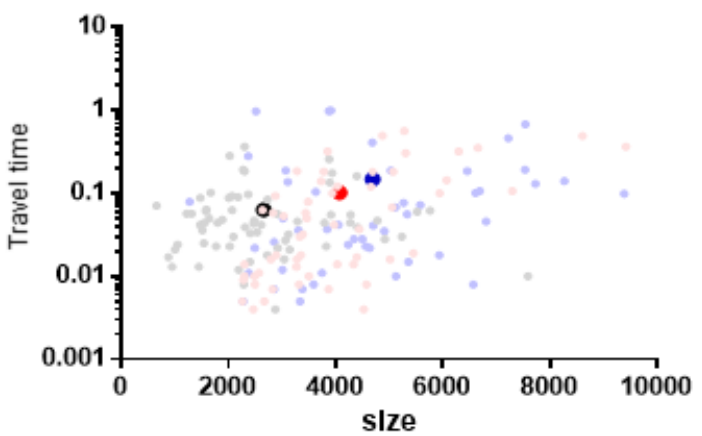

G

\begin{tabular}{|c|c|c|c|c|}
\hline \multirow{4}{*}{ Times } & $\begin{array}{c}\text { K-L } \\
\text { Divergence }\end{array}$ & Moruloid & Blastuloid & $\begin{array}{c}\text { BM-ve } \\
\text { blastuloid }\end{array}$ \\
\hline \multirow{4}{*}{ Entry time } & Moruloid & - & 1.5581 & 0.3873 \\
\cline { 2 - 5 } & Blastuloid & 2.1994 & - & 1.0183 \\
\cline { 2 - 5 } & BM-ve & 0.5370 & 2.2502 & - \\
\hline \multirow{3}{*}{ Travel time } & Moruloid & - & 1.217 & 0.1979 \\
\cline { 2 - 5 } & Blastuloid & 1.0663 & - & 0.9022 \\
\cline { 2 - 5 } & BM-ve & 0.3140 & 1.6839 & - \\
\hline
\end{tabular}


Figure 2: Blastuloid spheroids travel through the channel faster than moruloid spheroids. (A, B) Schematic representation of two analyzed time metrics entry time (A) and travel time (B). (C, D) Scatter graphs showing entry times (C) and travel times (D) for moruloid, blastuloid and BM-removed blastuloid spheroids. One-way ANOVA with post hoc Tukey's comparison used to compute statistical significance $(p=0.002, p=0.02)$ (see also Fig. S5 B). (E, F) Scatter plots showing correlations of entry times (E) and travel time (F) for moruloid (light red dots), blastuloid (light black dots) and BM-removed blastuloid spheroids (light blue dots) with centroids for the three types shown as single big red, black, and blue dots respectively (see also Fig. S5 B). (G) Table showing K-L divergence values for correlation between entry and travel times and sizes for moruloid, blastuloid and BMremoved blastuloid spheroids (see also Fig. S6).
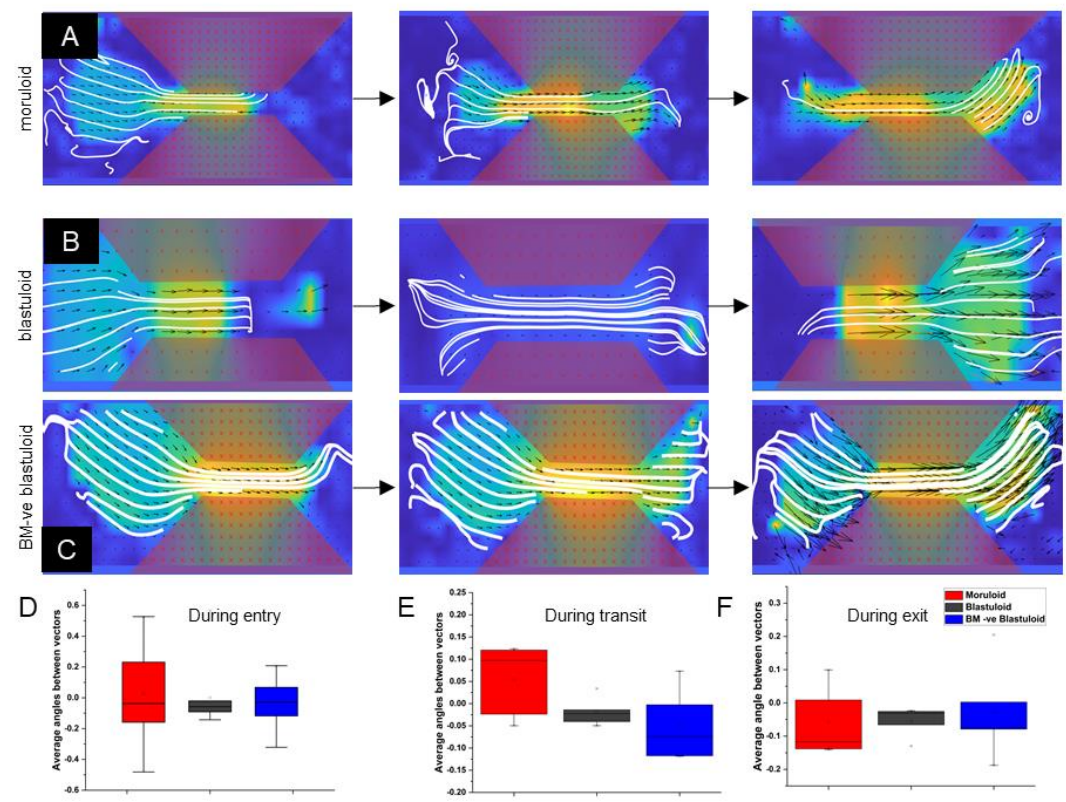

Figure 3: Intercellular rearrangement within blastuloid spheroids during constrictive traversal is minimal. (A-C) Velocity gradient plots obtained from performing particle image velocimetry showing streamlines (white) and velocity vectors (black) for (A) moruloid (B) blastuloid and (C) BM-removed blastuloid spheroids. Red portions represent the mask (areas not analyzed for flow). (D-F) Box plots showing variation in the angles between the vectors, obtained using particle image velocimetry for moruloid, blastuloid and BM-removed blastuloid spheroids, at the time of entry into the channel (D), within the channel $(E)$ and during exit from the channel $(F)$. 

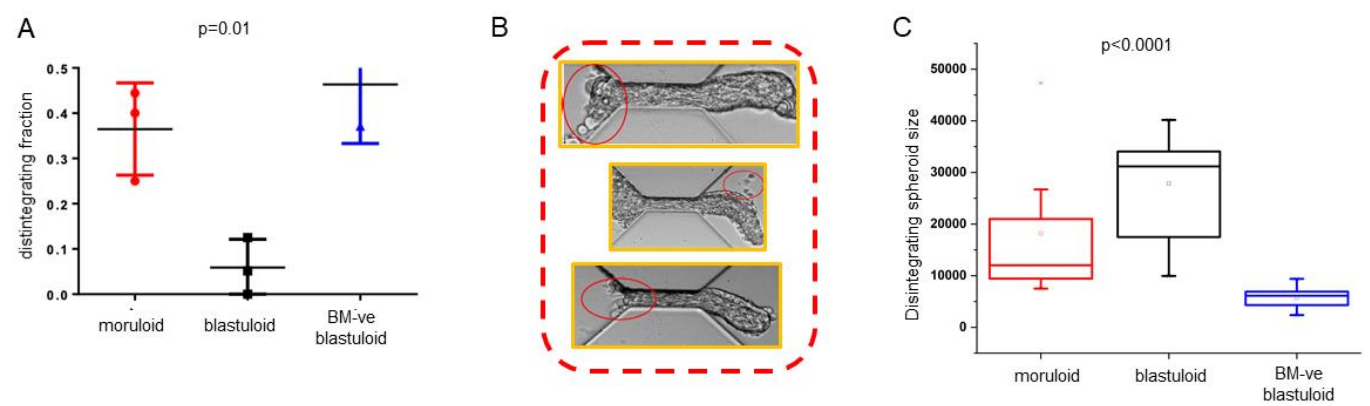

Figure 4: Blastuloid spheroids are resilient to cell detachment. (A) Dot plots showing the proportion of moruloid spheroids, blastuloid spheroids, and blastuloid spheroids with Type IV collagenase treatment undergoing cell detachment $(p=0.01)$. (B) Representative images showing cell detachment and disintegration in moruloid spheroids as they traverse the constrictive channel (see also Video S9, S10 ans S11). (C) Box plot showing the size of moruloid, blastuloid and collagenase treated blastuloid spheroids showing cell detachment and disintegration. One-way ANOVA with post hoc Tukey's comparison used to compute statistical significance of area values taken from all 3 biologically independent experiments $(p<0.0001)$. Error bars represent mean $+/-$ SEM. Scale bars $=50 \mu \mathrm{m}$. 


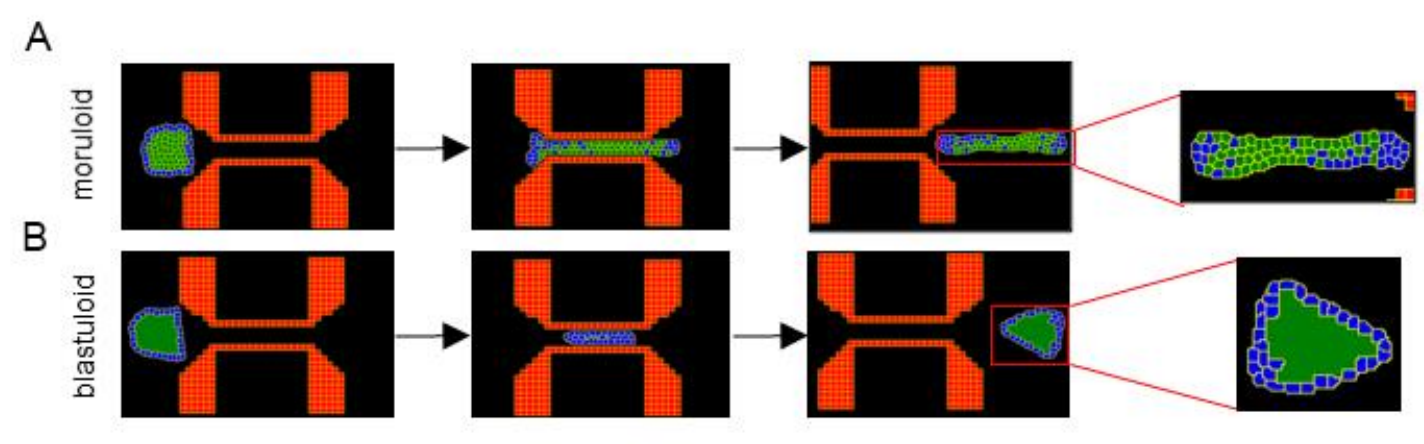

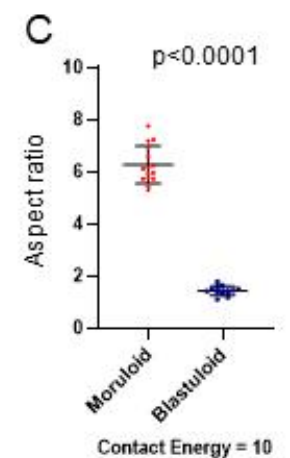
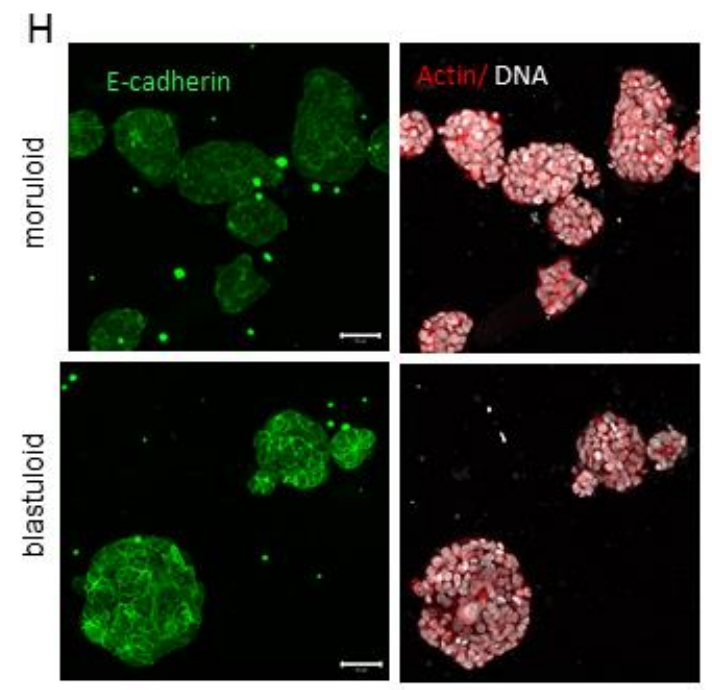
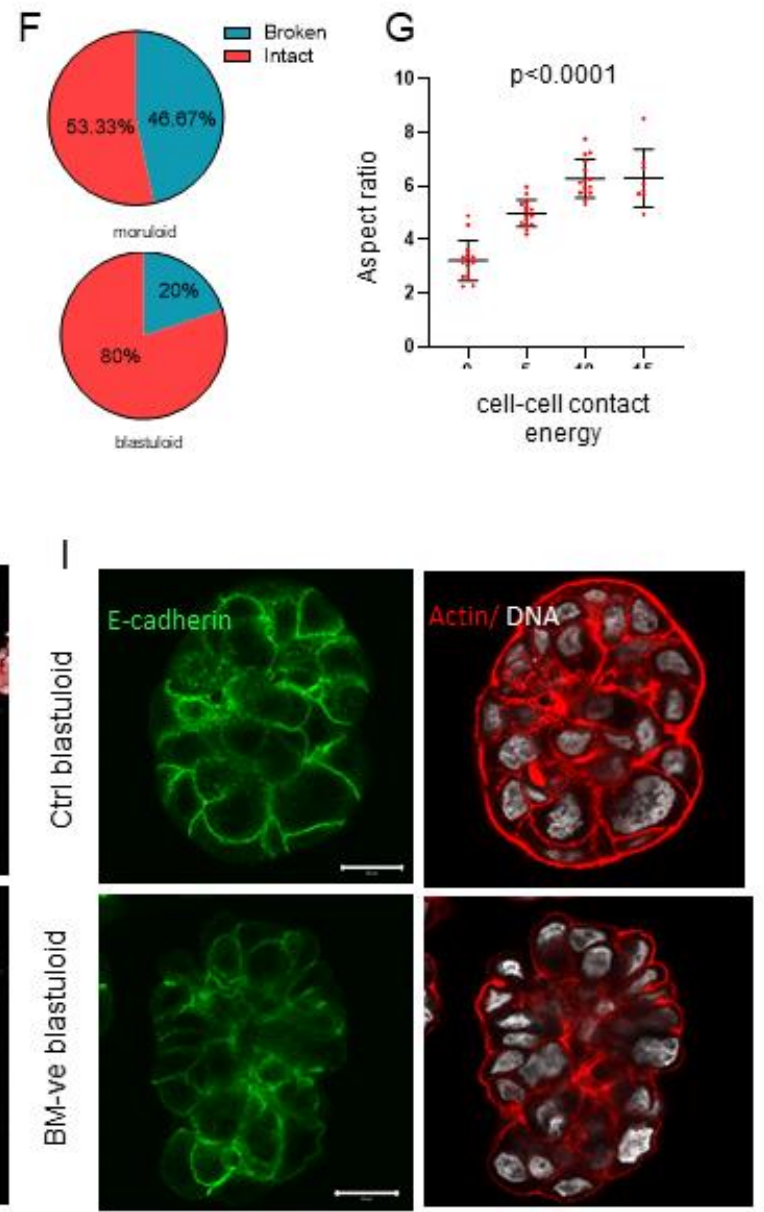

Figure 5: Higher intercellular adhesion drives jamming behavior in ovarian cancer blastuloid spheroids. (A, B) Snapshots of Compucell3D simulations of (A) digital moruloid and (B) blastuloid spheroids traversing through a spatially constrictive channel representing time points when the spheroids enter the channel (left) inside the channel (middle) and during exit from the channel (right). Insets show the intercellular arrangement within the digital spheroids upon exit (see also Video S12 and S13). (C) Scatter graph showing the difference between aspect ratios of digital moruloid and blastuloid spheroids 
upon exit from the channel normalized to the same at entry. Unpaired Student's t-test used to compute statistical significance $(p<0.0001)$. (D, E) Snapshot of a Compucell3D simulation of a digital (D) moruloid and (E) blastuloid spheroid showing disintegration and cell detachment upon traversal (see also Video S14 and S15). (F) Pie-charts representing fraction of disintegration upon traversal. (G) Aspect ratio of digital moruloid spheroids with contact energy. One-way ANOVA with post hoc Tukey's comparison used to compute statistical significance $(p<0.0001)$. $(H)$ Laser confocal micrographs of moruloid (top) and blastuloid (bottom) OVCAR3 spheroids showing maximum intensity projection of the fluorescence values representing E-cadherin (green) and counterstaining for F-actin (phalloidin; red) and DNA (DAPI; white). (I) Laser confocal micrographs of blastuloid OVCAR3 spheroids (untreated control top) and upon Type IV collagenase treatment (bottom) showing middle stack of the fluorescence values representing E-cadherin (red) and counterstaining for F-actin (phalloidin; green). Error bars represent mean+/- SEM. Scale bars $=50 \mu \mathrm{m}$ and $20 \mu \mathrm{m}$ respectively (see also Fig. S3). 


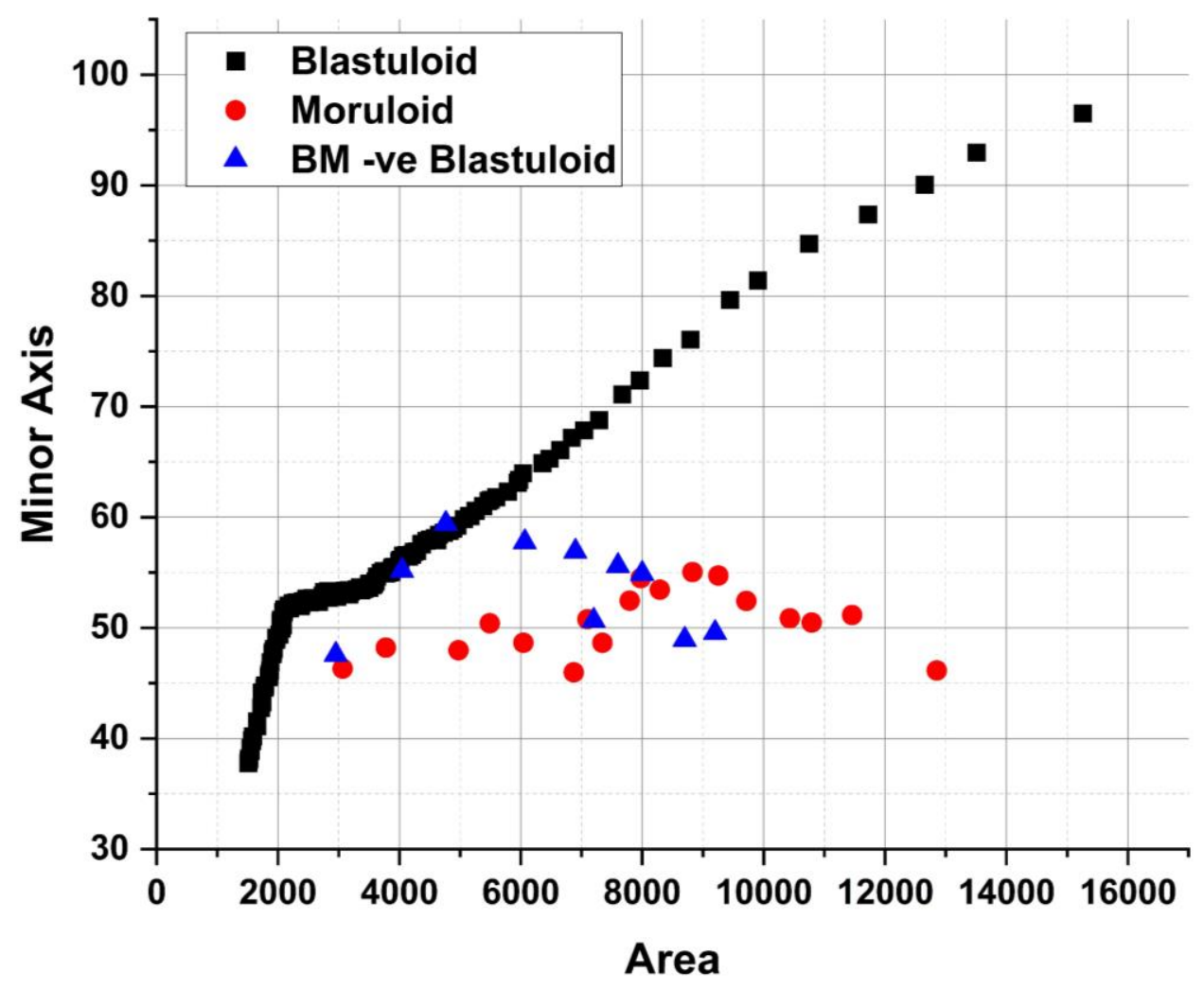

Supplementary figure 1: Scatter plot showing minor axis evolution with area of the exiting cluster for a representative moruloid, blastuloid and BM -ve blastuloid spheroid. 

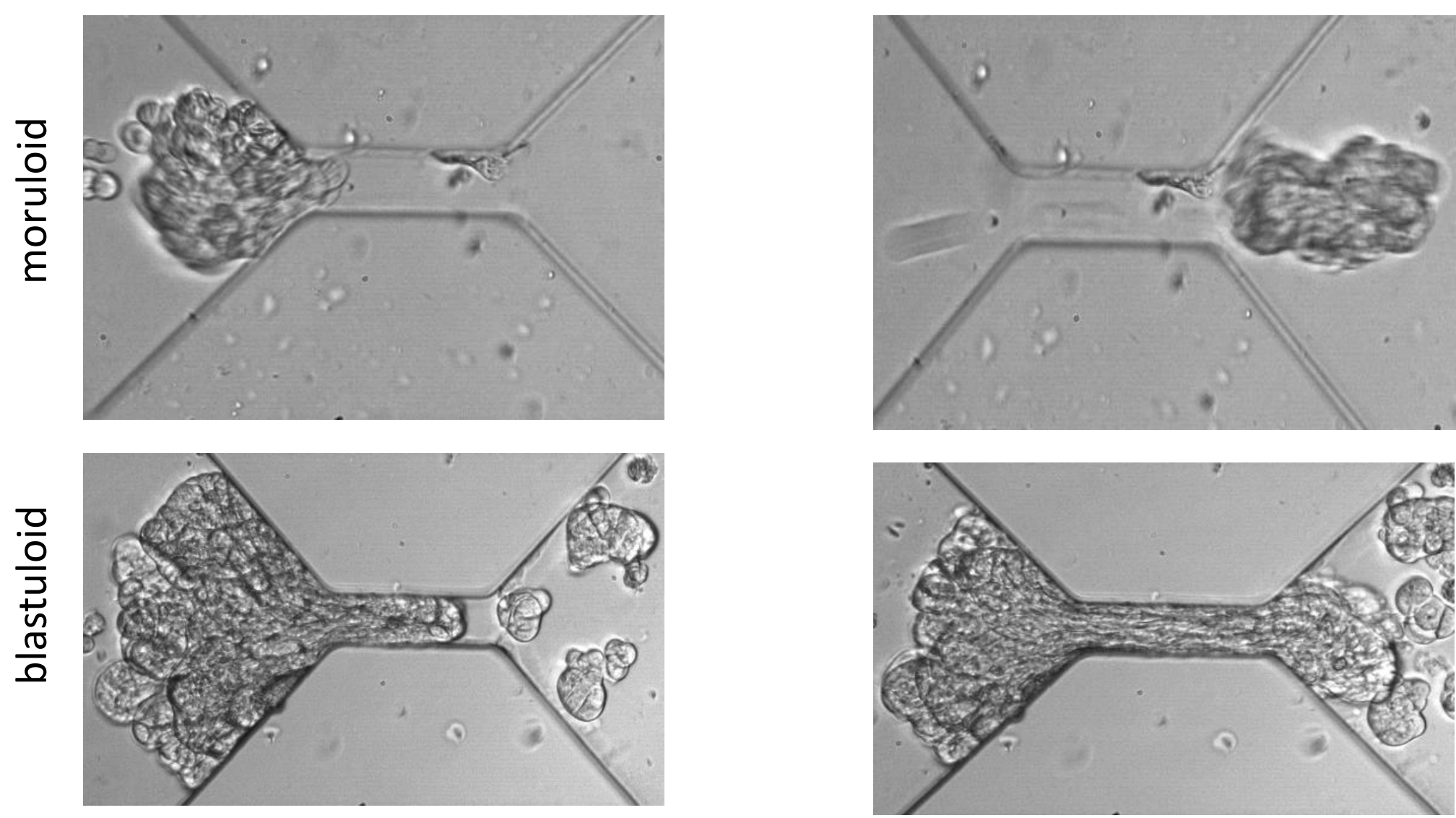

Supplementary figure 2: Snapshots showing exit appearance of moruloid and blastuloid morphology in G1M2 cell line. 

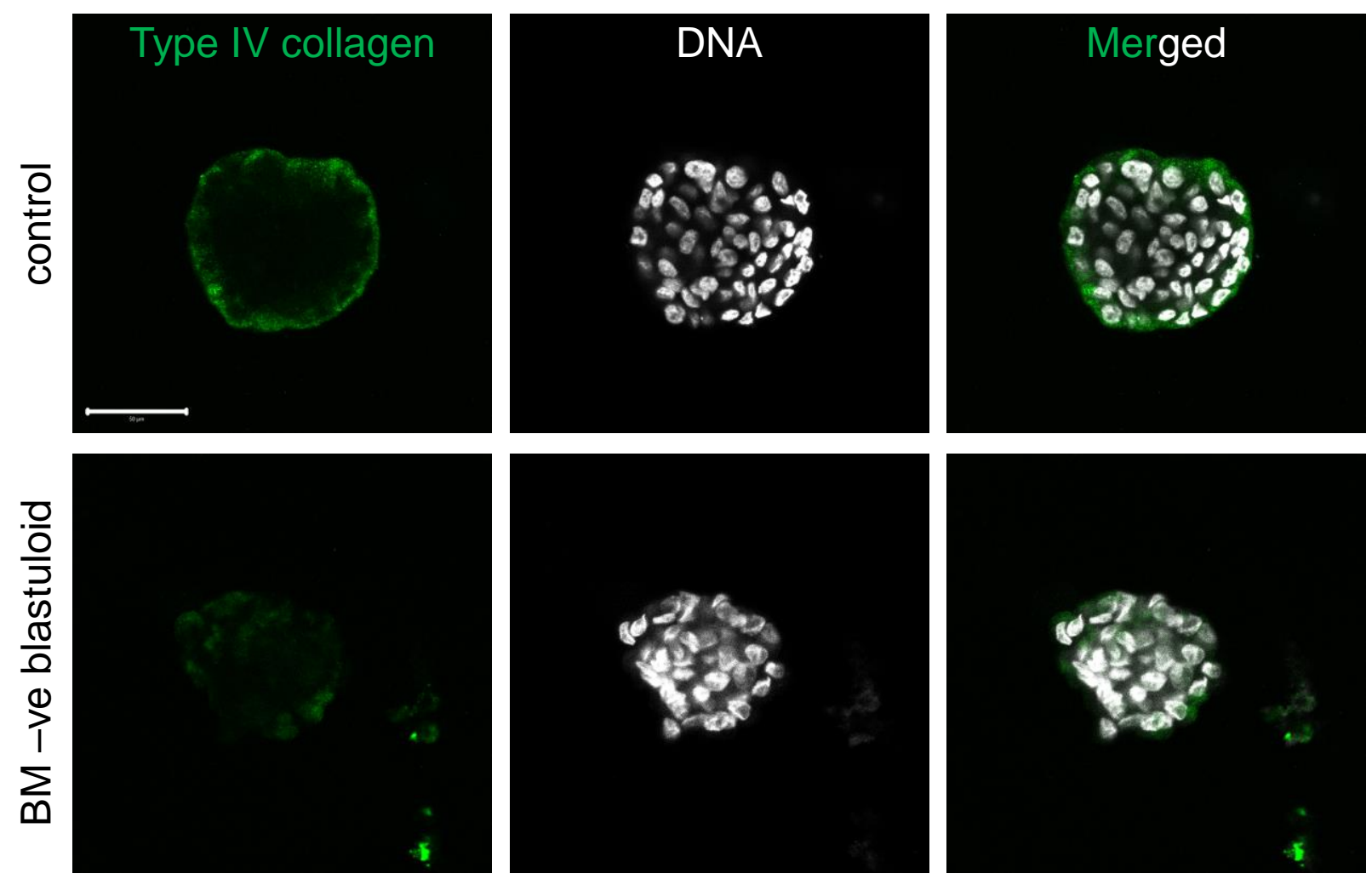

Supplementary figure 3: Laser confocal micrographs of blastuloid OVCAR3 spheroids (top) and BM -ve blastuloid OVCAR3 spheroids (bottom) showing mid stack of the fluorescence values representing Type IV collagen (green) and counterstaining for DNA (DAPI; white). Scale bars $=50 \mu \mathrm{m}$. 


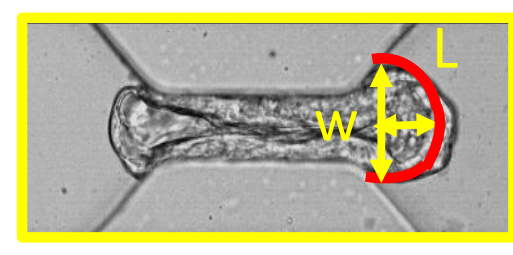

B
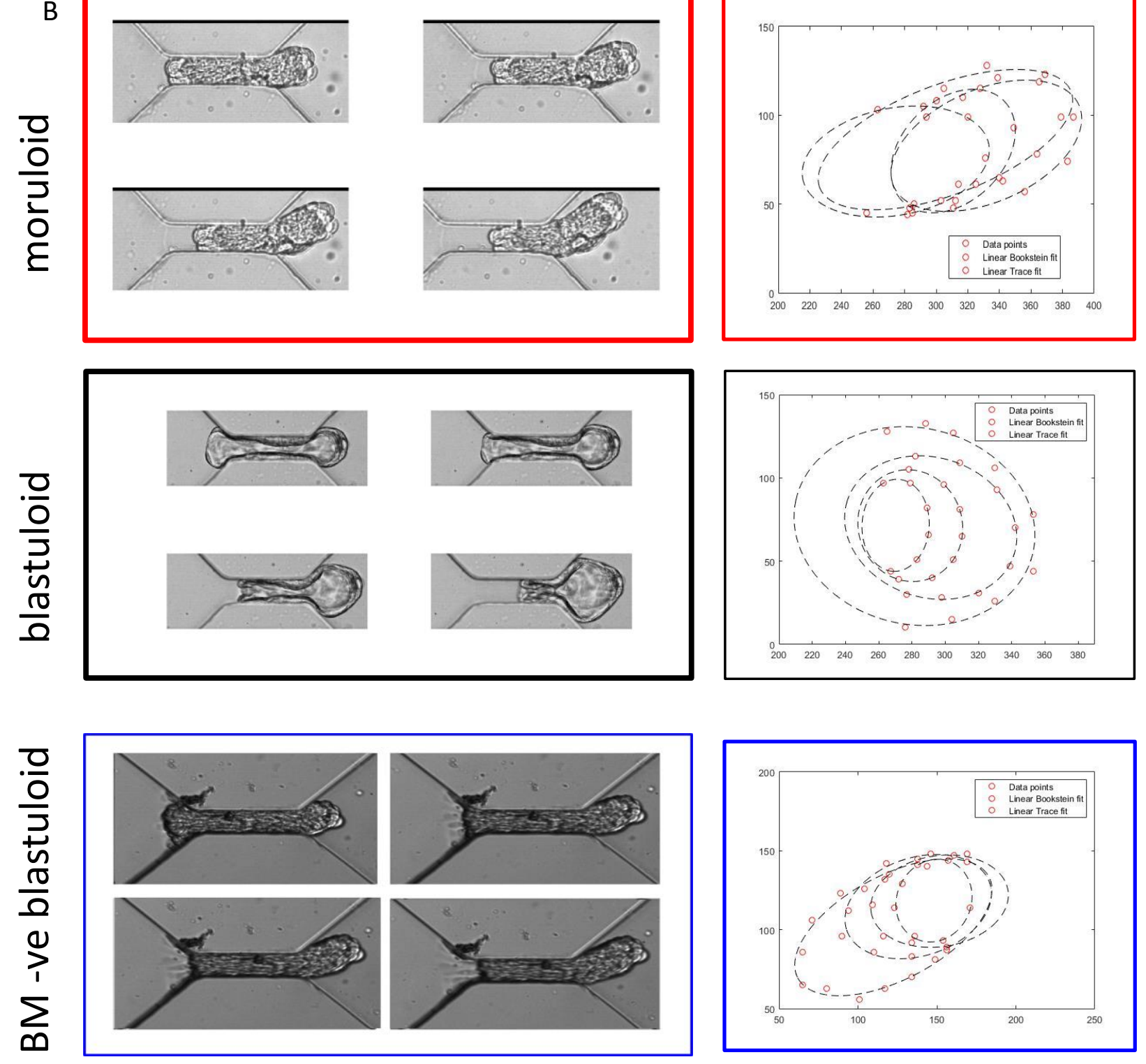

C

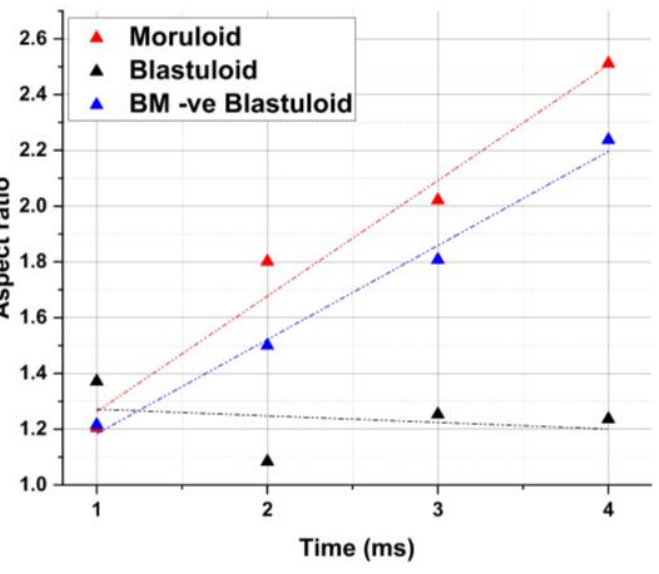

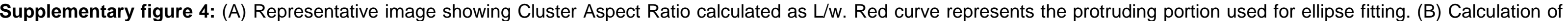

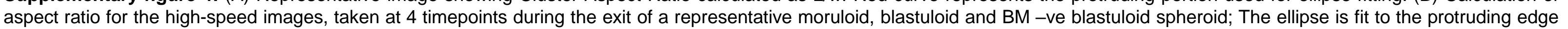

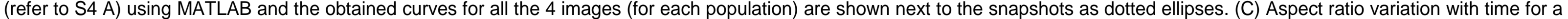

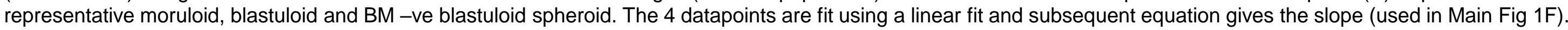




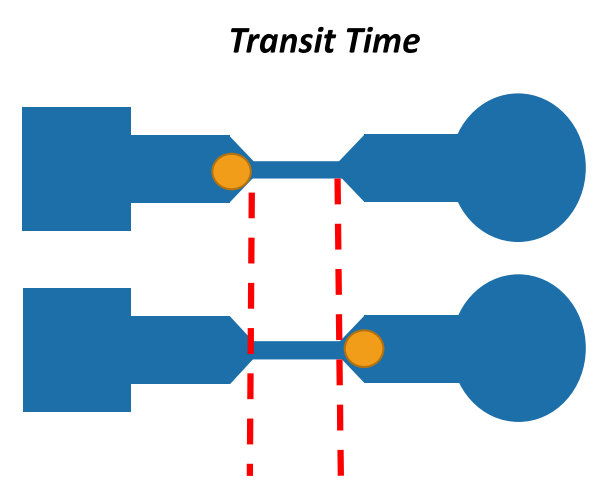

$\mathrm{dt}=\operatorname{tr} 2-\operatorname{tr} 1$

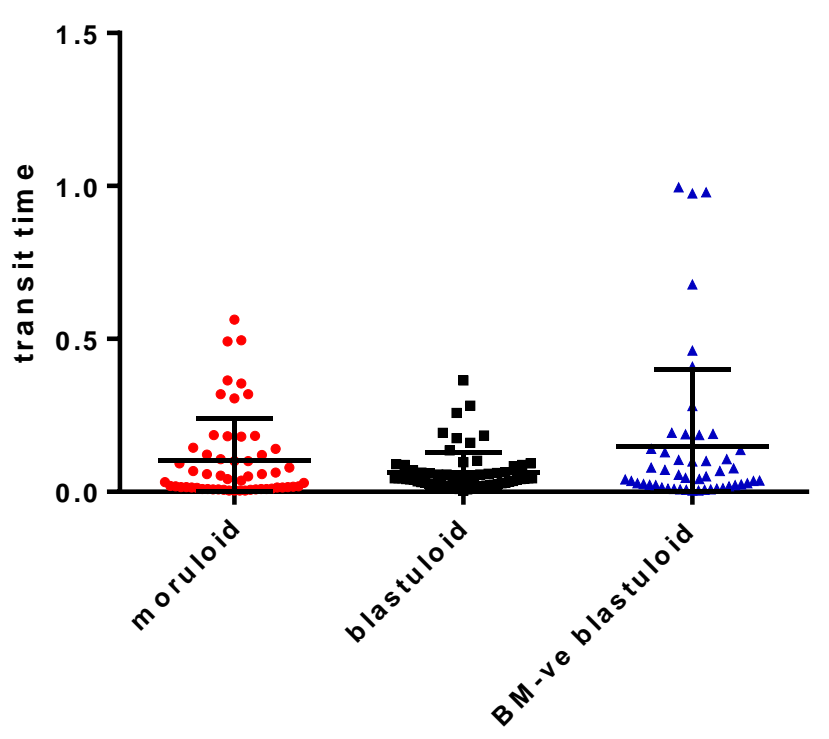

C

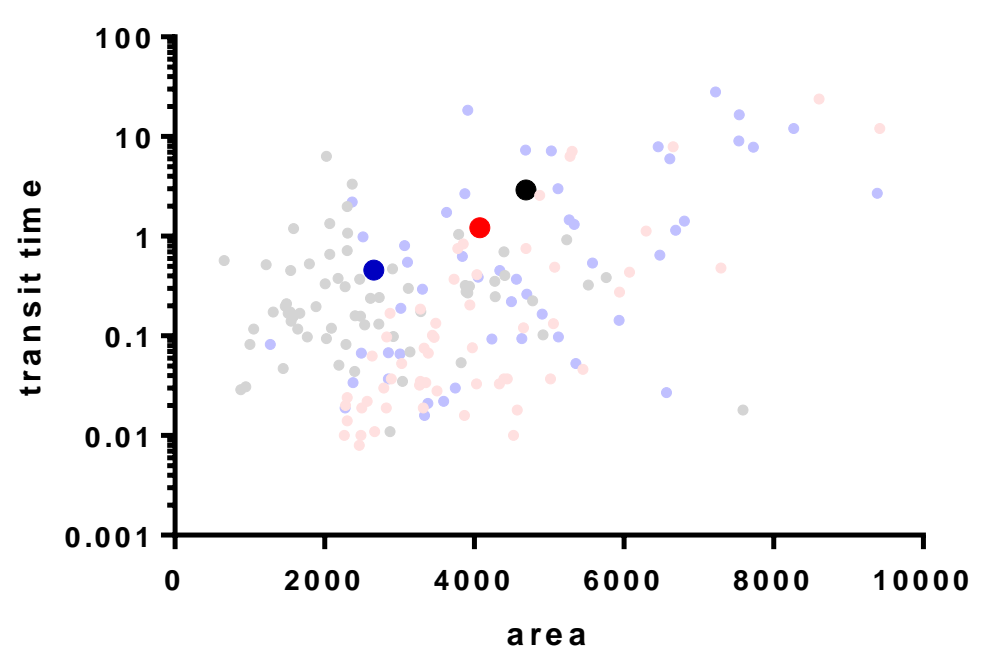

Supplementary figure 5: (A) Schematic representation of time metric: transit time; defined as the time taken for the leading edge of the cluster to just enter and the trailing edge to just exit the channel. (B) Scatter graphs showing transit times for moruloid, blastuloid and BM-removed blastuloid spheroids. (C) Scatter plots showing correlations of transit times for moruloid (light red dots), blastuloid (light black dots) and BM-removed blastuloid spheroids (light blue dots) with centroids for the three types shown as single big red, black, and blue dots respectively. One-way ANOVA with post hoc Tukey's comparison used to compute statistical significance. 
A

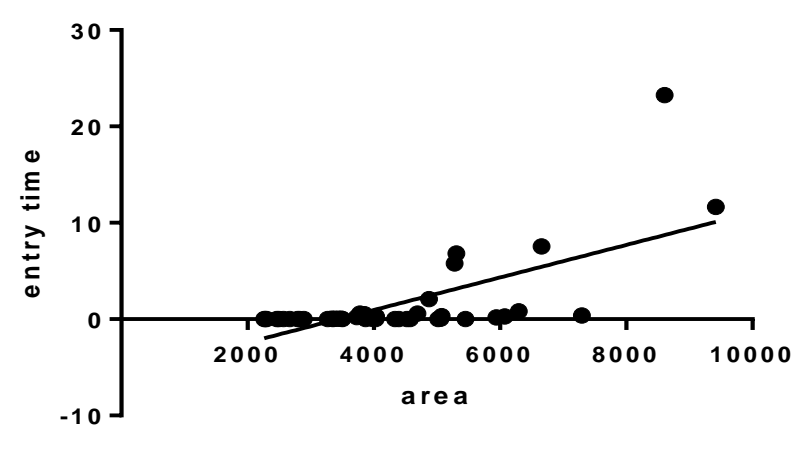

moruloid

B

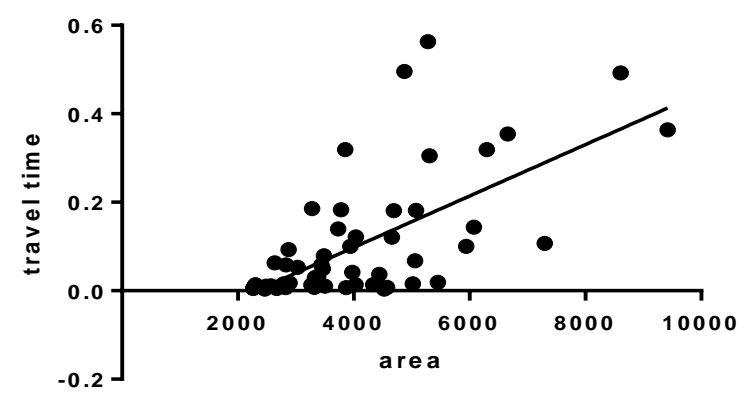

C

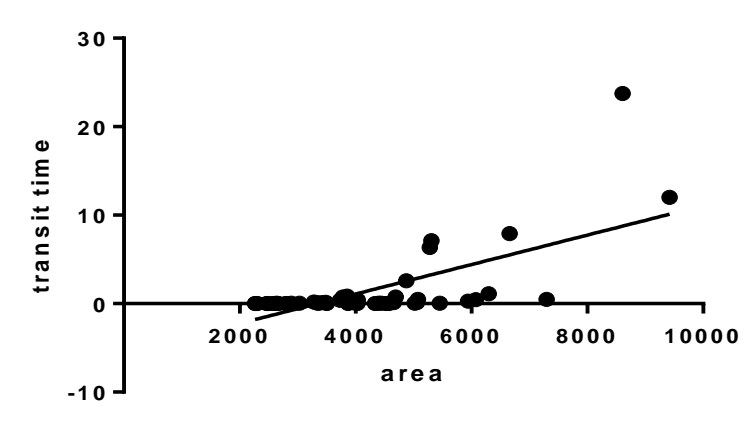

blastuloid
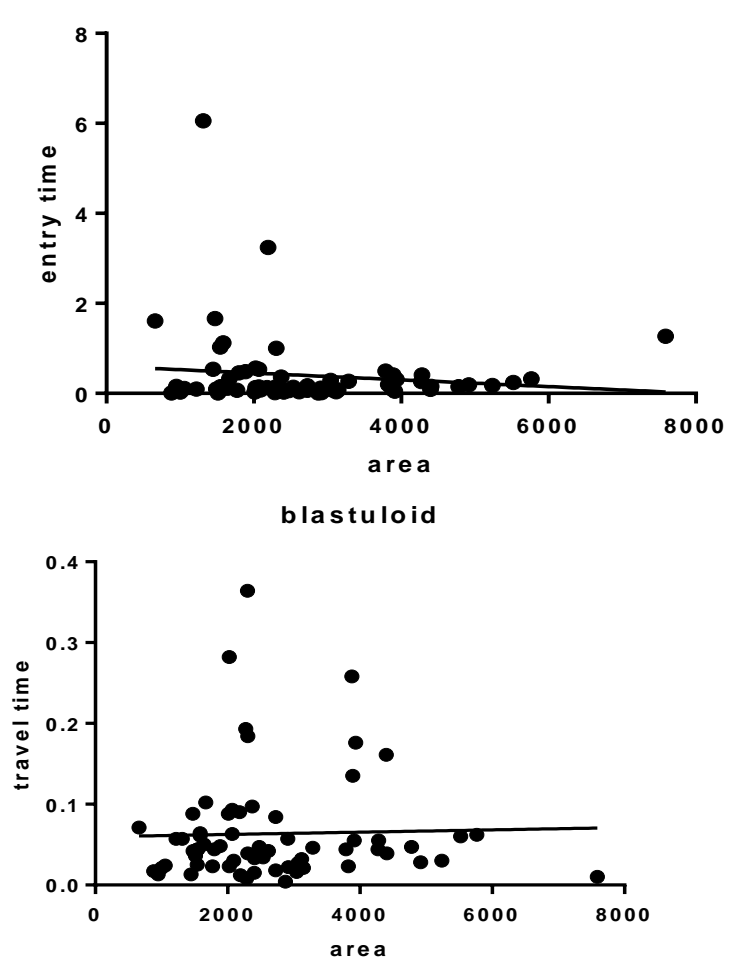

blastuloid

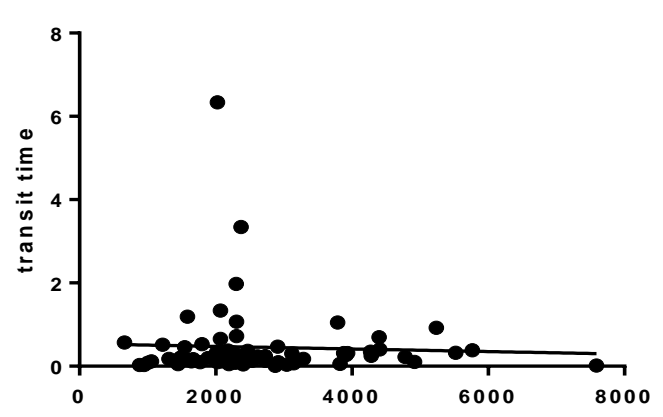

BM removed blastuloid

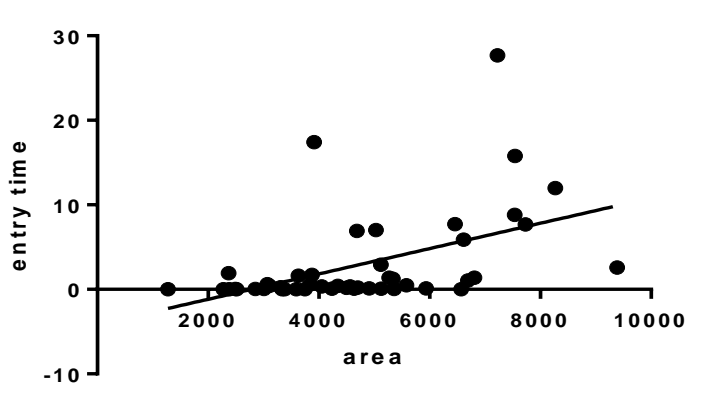

BM removed blastuloid

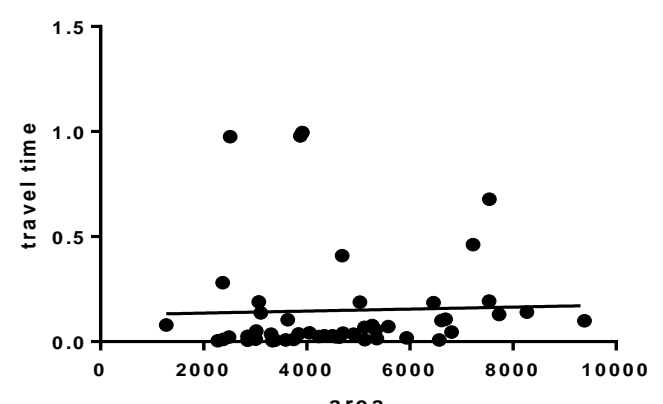

BM removed blastuloid

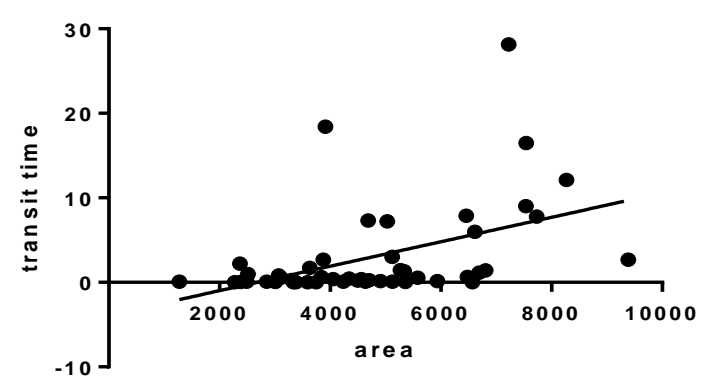

Supplementary figure 6: Scatter graphs showing variation of (A) entry (B) travel and (C) transit times for moruloid, blastuloid and BM-removed blastuloid spheroids 


\section{Supplementary Video Legends:}

Video S1: Transit of OVCAR3 moruloid spheroid through micro-channel.

Video S2: Transit of OVCAR3 blastuloid spheroid through micro-channel.

Video S3: Transit of G1M2 moruloid spheroid through micro-channel.

Video S4: Transit of G1M2 blastuloid spheroid through micro-channel.

Video S5: Transit of OVCAR3 BM-ve blastuloid spheroid through micro-channel.

Video S6: Recovery of moruloid spheroid on exiting the micro-channel.

Video S7: Recovery of blastuloid spheroid on exiting the micro-channel.

Video S8: Recovery of BM -ve blastuloid spheroid on exiting the micro-channel.

Video S9-11: Cell detachment of moruloid spheroid during transit.

Video S12: Sustained deformation and intercellular rearrangement in digital moruloid spheroid.

Video S13: Recovery of digital blastuloid spheroids upon exit.

Video S14: Cell detachment and disintegration in digital moruloid spheroid.

Video S15: Cell detachment and disintegration in digital blastuloid spheroid. 